\title{
Reducing dynamin 2 expression rescues X-linked centronuclear myopathy
}

\author{
Belinda S. Cowling, ${ }^{1}$ Thierry Chevremont, ${ }^{1}$ Ivana Prokic, ${ }^{1}$ Christine Kretz, ${ }^{1}$ \\ Arnaud Ferry, ${ }^{2,3,4,5,6}$ Catherine Coirault, ${ }^{2,3,4,5}$ Olga Koutsopoulos, ${ }^{1}$ Vincent Laugel, ${ }^{7}$
} Norma B. Romero, ${ }^{2,3,8,9,10}$ and Jocelyn Laporte ${ }^{1}$

\begin{abstract}
1Department of Translational Medicine and Neurogenetics, Institut de Génétique et de Biologie Moléculaire et Cellulaire (IGBMC), INSERM, U964, CNRS, UMR7104, Université de Strasbourg, Collège de France, Chaire de Génétique Humaine, Illkirch, France. ${ }^{2}$ INSERM UMR974, F-75013, Paris, France. ${ }^{3}$ CNRS, UMR7215, F-75013, Paris, France. ${ }^{4}$ Sorbonne Universités, Université Pierre et Marie Curie-Paris 6 , UM76, F-75005, Paris France. ${ }^{5}$ Institut de Myologie, F-75013, Paris France. ${ }^{6}$ Université Paris Descartes, Paris Sorbonne Cité, F-75006, Paris, France. ${ }^{7}$ Department of Pediatrics, Strasbourg-Hautepierre University Hospital, Strasbourg, France. ${ }^{8}$ Unité de Morphologie Neuromusculaire, Institut de Myologie, GHU La Pitié-Salpêtrière, Paris, France. 9Université Pierre et Marie Curie-Paris 6, UM76, F-75013, Paris, France. ${ }^{10}$ Centre de Référence de Pathologie Neuromusculaire Paris-Est, Groupe Hospitalier La Pitié-Salpêtrière, Paris, France.
\end{abstract}

\begin{abstract}
Centronuclear myopathies (CNM) are congenital disorders associated with muscle weakness and abnormally located nuclei in skeletal muscle. An autosomal dominant form of CNM results from mutations in the gene encoding dynamin 2 (DNM2), and loss-of-function mutations in the gene encoding myotubularin (MTM1) result in X-linked CNM (XLCNM, also called myotubular myopathy), which promotes severe neonatal hypotonia and early death. Currently, no effective treatments exist for XLCNM. Here, we found increased DNM2 levels in XLCNM patients and a mouse model of XLCNM $\left(\mathrm{Mtm}^{-/ y}\right)$. Generation of $\mathrm{Mtm}^{-/ y}$ mice that were heterozygous for $D n m 2$ revealed that reduction of DNM2 in XLCNM mice restored life span, whole-body strength, and diaphragm function and increased muscle strength. Additionally, classic CNM-associated histological features, including fiber atrophy and nuclei mispositioning, were absent or reduced. Ultrastructural analysis revealed improvement of sarcomere organization and triad structures. Skeletal muscle-specific decrease of $D n m 2$ during embryogenesis or in young mice after disease onset revealed that the rescue associated with downregulation of $\mathrm{Dnm} 2$ is cell autonomous and is able to stop and potentially revert XLCNM progression. These data indicate that MTM1 and DNM2 regulate muscle organization and force through a common pathway. Furthermore, despite DNM2 being a key mechanoenzyme, its reduction is beneficial for XLCNM and represents a potential therapeutic approach for patients.
\end{abstract}

\section{Introduction}

Centronuclear myopathies (CNM) are a group of congenital myopathies characterized by muscle weakness, fiber atrophy, predominance of type I fibers, and increased centralization of nuclei not secondary to muscle regeneration $(1,2)$. Three main forms of CNM have been characterized: X-linked CNM (XLCNM; OMIM 310400), due to mutations in the phosphoinositides phosphatase myotubularin (MTM1) (3); autosomal recessive CNM (ARCNM, OMIM 255200), caused by mutations in the membrane remodeling protein amphiphysin 2 (BIN1) (4), and autosomal dominant CNM (ADCNM, OMIM 160150), due to mutations in dynamin 2 (DNM2) (5). The relationship between the implicated genes in muscle is not known, and potent therapeutic approaches are lacking.

XLCNM, also called myotubular myopathy, is the most common and severe form of CNM, with neonatal onset and a poor prognosis (1). To date, more than 200 different mutations in MTM1 have been reported in about 450 families, most of which lead to a strong reduction of MTM1 protein (6-9). Mtm1-knockout mice or mice with knockin of a patient mutation both recapitulate the CNM phenotype with classical histological features including abnormal organelle positioning, mislocalization of nuclei, and muscle atrophy, associated with a corresponding reduction in muscle strength (10-12). A defect in triad structures associated with abnormal excitation-contraction coupling has been reported in several animal

Conflict of interest: The authors have declared that no conflict of interest exists. Citation for this article: J Clin Invest. 2014;124(3):1350-1363. doi:10.1172/JCI71206. models and patients with various forms of CNM (13-15), identifying a common defect in all CNM forms. This is consistent with a proposed role of MTM1 in the regulation of phosphoinositides level on the sarcoplasmic reticulum component of the triads $(14,16)$.

Dynamins are large GTPase proteins that play important roles in membrane trafficking and endocytosis (17-20) and in actin cytoskeleton assembly (19-21). Dynamins can form polymerized rings around membrane tubules (22) and are involved in membrane fission events $(20,23,24)$, providing a paradigm of a mechanoenzyme. Three dynamins have been identified in humans: dynamin 1 , expressed mainly in neurons, dynamin 3, expressed predominantly in brain and testis, and DNM2, which is ubiquitously expressed. Different heterozygous DNM2 mutations have been identified in tissue-specific diseases; ADCNM affecting skeletal muscle (5) and autosomal dominant Charcot-Marie-Tooth (CMTDIB, OMIM 606482) peripheral neuropathy (25).

Recent biochemical studies have indicated that some CNMcausing DNM2 mutations increase dynamin oligomer stability and GTPase activity $(26,27)$. This was supported in vivo by either knockin or overexpression of the most common CNM-DNM2 patient mutation in mice, which induced CNM-like features at adulthood $(28,29)$, indicating that the disease is not due to haploinsufficiency. Overexpression of WT DNM2 also caused similar CNM-like perturbation to the muscle, albeit to a lesser extent $(28,30)$.

The main goal of this study was to validate a rescue approach for XLCNM. Due to their common implication in CNM, we suspected that MTM1 and DNM2 might function in a common pathway, 
A

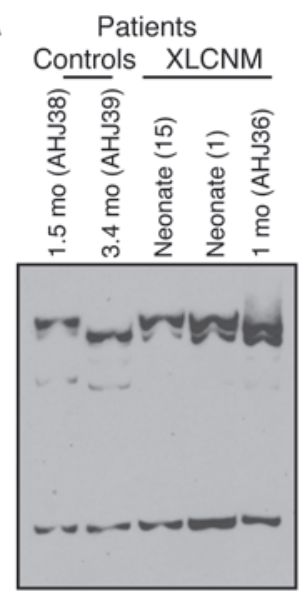

E

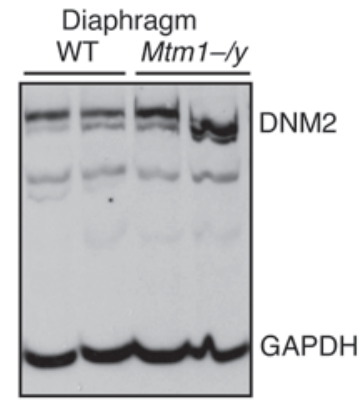

B

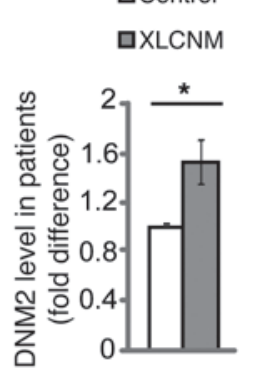

GAPDH

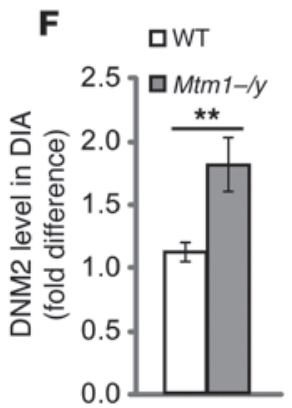

C

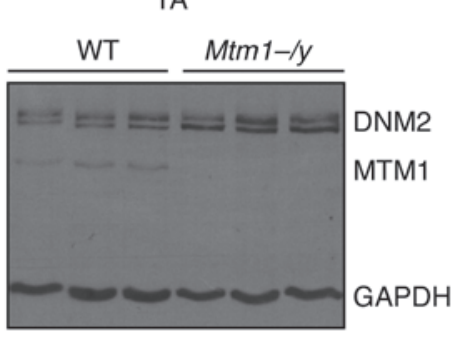

G
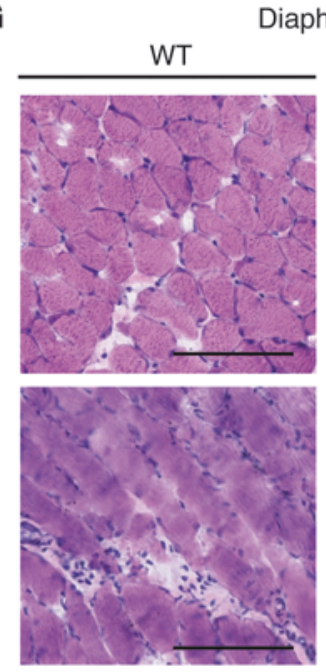

D

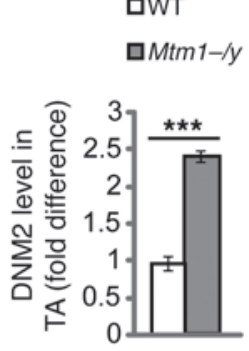

\section{Figure 1}

DNM2 levels in XLCNM. (A) Representative Western blot of XLCNM patient muscle lysates for DNM2, MTM1, and GAPDH loading control. (B) Relative level of DNM2 protein expression determined by densitometry, standardized to GAPDH. $n=5$ patients. TA (C) and diaphragm (E) skeletal muscle lysates from 5-week-old WT and $\mathrm{Mtm}^{-1 / y}$ mice were immunoblotted for DNM2 and GAPDH. Relative levels of DNM2 protein determined by densitometry of DNM2 immunoreactive polypeptides, standardized to GAPDH, for TA (D) and diaphragm (F). DNM2 expression is represented as fold difference from WT lysate. $n=4$ mice. (G) Diaphragm muscle sections stained for H\&E. Scale bars: $100 \mu \mathrm{m}$. All graphs depict mean \pm SEM. ${ }^{\star} P<0.05 ;{ }^{* \star} P<0.01 ;{ }^{* \star *} P<0.001$.

where either MTM1 loss-of-function or DNM2 gain-of-function lead to the CNM phenotype. We hypothesized that MTM1 might be a negative regulator of DNM2 function in muscle and that reduced DNM2 expression in an XLCNM model may rescue the XLCNM phenotype. To test this hypothesis, we produced $\mathrm{Mtm}^{-/ y}$ mice that were heterozygous for DNM2 (Mtm1 $\left.1^{-/ y} \mathrm{Dnm} 2^{+/-}\right)$. We found that reducing DNM2 expression in $\mathrm{Mtm}^{-1 / y}$ mice is sufficient to rescue the early XLCNM lethality and most features of the disease. We also showed that reducing DNM2 expression only in muscle from embryogenesis, or even after the onset of the disease, was sufficient to increase the life span of $\mathrm{Mtm}^{-1 / y}$ mice. Finally we provide what we believe is the first evidence that MTM1 is a regulator of DNM2 in skeletal muscle and propose a rationale disease for treatment.

\section{Results}

Characterization of Dnm2 heterozygous (Dnm2 $2^{+-}$) mice. Constitutive knockout of Dnm2 was previously shown to be lethal early during embryogenesis (31). We created $D n m 2^{--}$mice by targeting exon 8 (Supplemental Figure 1; supplemental material available online with this article; doi:10.1172/JCI71206DS1). From 100 pups, we did not identify any $D n m 2^{-/-}$mice, confirming that $D n m 2^{-/-}$is embryonically lethal. $D n m 2^{+/-}$pups were identified at expected Mendelian ratios and were further analyzed under the EUMODIC phenotyping program (32). Basic blood chemistry tests indicated no difference between WT and $\mathrm{Dnm}^{+} \mathrm{H}^{+-}$mice for urea (indicating normal kidney function), calcium (implying osmotic homeostasis), and total cholesterol (suggesting absence of cardiovascular disease) levels (Supplemental Figure 2A). Normal ECG measurements suggested unaltered electrical activity in the heart (Supplemental Figure 2B). Overall, there was no difference in body weight (Supplemental Figure 2C) or in lean tissue or fat content between WT and $D n m 2^{+/-}$mice (Supplemental Figure 2D). An electromyography test for basic muscle function revealed no difference in single nerve conduction velocity (Supplemental Figure 2E). Tibialis anterior (TA) muscle mass was similar between WT and $D n m 2^{+/-}$ mice (Supplemental Figure 2F), and no difference in absolute or specific maximal force or fatigability of the TA was detected (Supplemental Figure 2, G-I), indicating overall that $D n m 2^{+/-}$mice are clinically and physiologically similar to WT mice, with no detectable difference in muscle function.

DNM2 levels in XLCNM. Before investigating the therapeutic potential of downregulation of DNM2 in XLCNM, we measured DNM2 protein levels by Western blot on XLCNM patient muscle lysates (Figure 1A). We identified a 1.5-fold increase in DNM2 protein expression from 5 neonatal XLCNM muscle biopsies tested when compared with control age-matched biopsies (Figure 1B). 
A

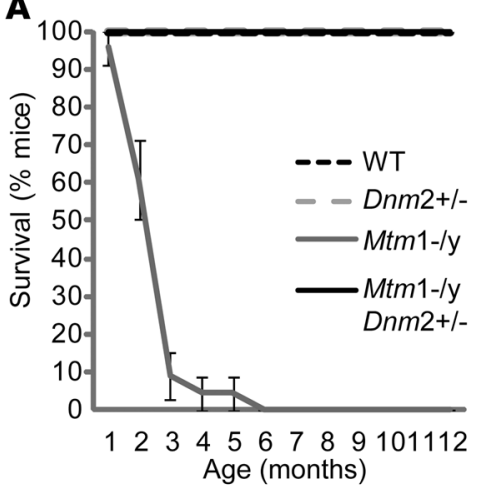

B

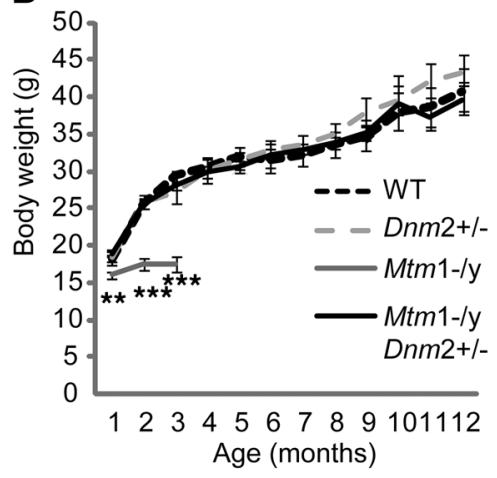

C

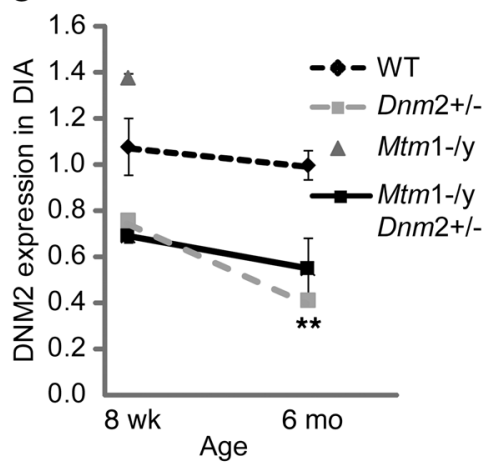

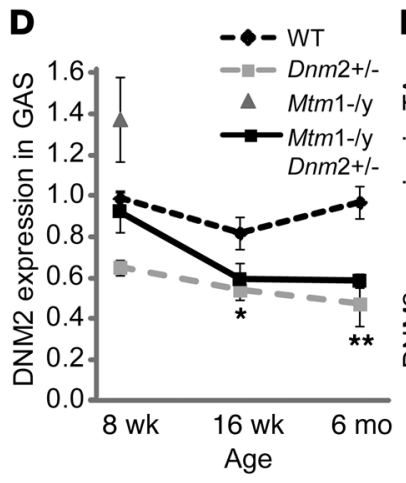

H

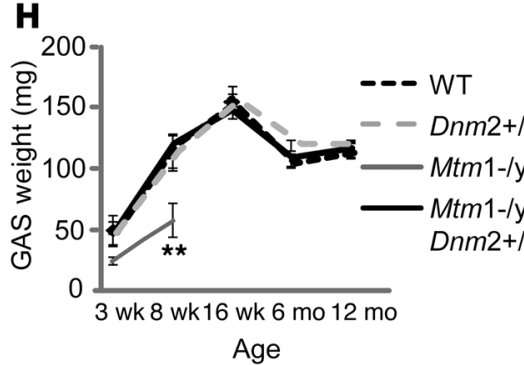

E

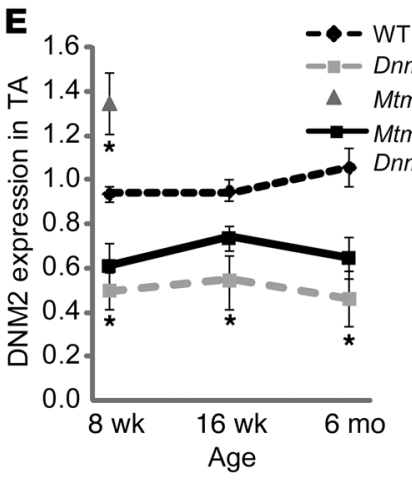

I
$\mathbf{F}$

G
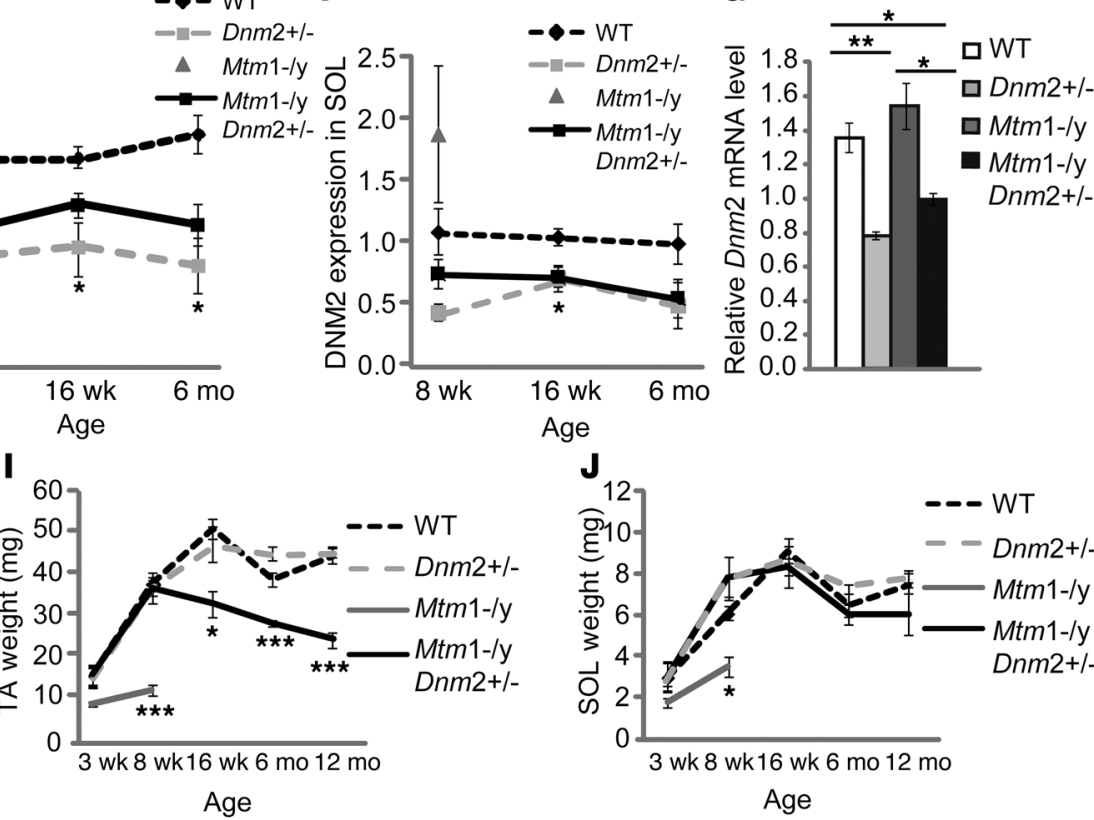

$-\bullet-W T$ - Dnm2+/$\Delta \mathrm{Mtm} 1-\mathrm{ly}$

$\rightarrow$ Mtm1-/y Dnm2+1-

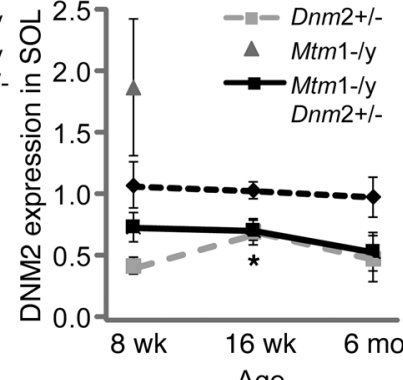

Age

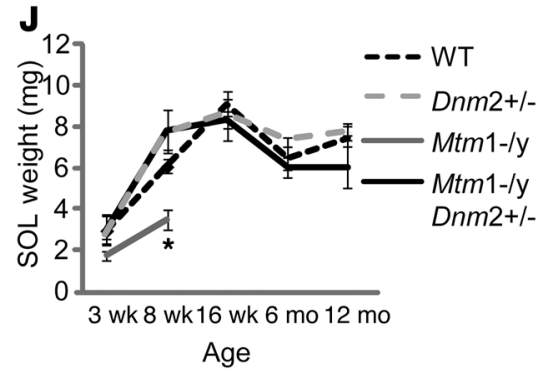

Figure 2

Reduced DNM2 expression greatly rescues the life span of $M t m 1^{-1 y}$ mice. (A) Life span of all mice, represented as percentage survival. All mice in groups WT, Dnm2 ${ }^{+-}$, and Mtm1-ly Dnm2+/- survived to at least 12 months. (B) Mouse whole-body weight. (C-F) Relative level of DNM2 protein was determined by densitometry of DNM2 standardized to GAPDH (blots on Supplemental Figure 5). DNM2 level is represented as fold difference from WT lysate. Expression was determined in 8-week-old, 16-week-old, and 6-month-old mice from diaphragm (DIA) (C), gastrocnemius (GAS) (D), TA (E), and soleus (SOL) (F) muscles. $n=2-8$ mice. (G) mRNA levels were quantified by qRT-PCR analysis, with DNM2 levels expressed relative to GAPDH. Graph represents 3 independent experiments. Gastrocnemius (H), TA (I), and soleus $(\mathbf{J})$ muscle weights $(n=5-13$ mice). All graphs depict mean \pm SEM. ${ }^{\star} P<0.05 ;{ }^{\star \star} P<0.01 ;{ }^{\star \star \star} P<0.001$. w, weeks of age; $\mathrm{m}$, months of age.

We then determined whether the increase in DNM2 expression was also observed in an animal model of XLCNM. In this study, we used $M t m 1^{-/ y}$ mice that faithfully reproduce XLCNM $(10,15)$. TA muscle lysates from 5-week-old Mtm $1^{-/ y}$ mice exhibited a significant increase in DNM2 levels compared with WT littermates (Figure 1, C and D), suggesting that increased DNM2 is linked to the XLCNM phenotype. An increase in DNM2 expression was also observed in the diaphragm (Figure 1, E and F), which appeared affected histologically, with more atrophic fibers containing mislocalized nuclei (Figure 1G). This finding suggests respiratory insufficiency as a cause of death in $\mathrm{Mtm}^{-/ y}$ mice, similar to what occurs in patients.

Reducing DNM2 expression greatly prolongs the life span of $\mathrm{Mtm}^{-1 /}$ mice. Reducing DNM2 at the genetic level to $50 \%$ in the $\mathrm{Dnm} 2^{+/-}$mice has no detectable clinical or physiological effects. To determine whether reducing DNM2 expression may rescue XLCNM due to MTM1 mutations, we crossed $\mathrm{Mtm}^{+/-}$mice with $\mathrm{Dnm} 2^{+/-}$mice to produce male offspring that were $M t m 1^{-/ y} \mathrm{Dnm} 2^{+/-}$. Most $M t m 1^{-/ y}$ mice died between 1 and 3 months of age as previously reported (10), whereas $100 \%$ of $\mathrm{Mtm}^{-1} \mathrm{Dnm}^{+/-}$mice survived for at least 1 year (Figure $2 \mathrm{~A}$ ), with no significant difference in body weight compared with WT mice (Figure 2B), indicating that reduced expression of DNM2 can rescue the early lethality observed in $\mathrm{Mtm}^{-1 / y}$ mice. $\mathrm{Mtm}^{-1 / y} \mathrm{Dnm} \mathrm{2}^{+/-}$ mice that were not sacrificed reached 2 years of age. At 8 weeks, when approximately $60 \%$ of $M t m 1^{-/ y}$ mice were still alive, $M t m 1^{-/ y}$ $D n m 2^{+/-}$mice were not distinguishable from WT mice upon general inspection, whereas $\mathrm{Mtm}^{-1} \mathrm{y}$ mice displayed a significant decrease in movement and activity (Supplemental Video 1).

We determined the level of DNM2 protein expression on lysates from several muscles at different ages. In the diaphragm, DNM2 levels were reduced approximately $50 \%$ in $\mathrm{Dnm} 2^{+/-}$and 
A

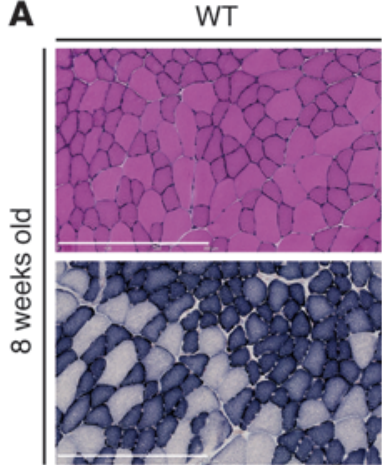

B

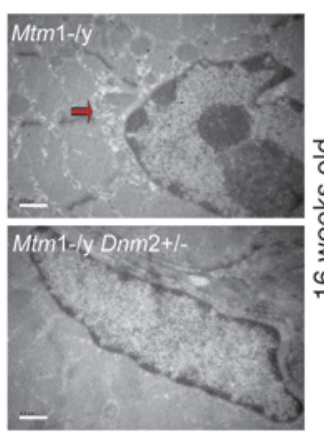

$8 w k$

D

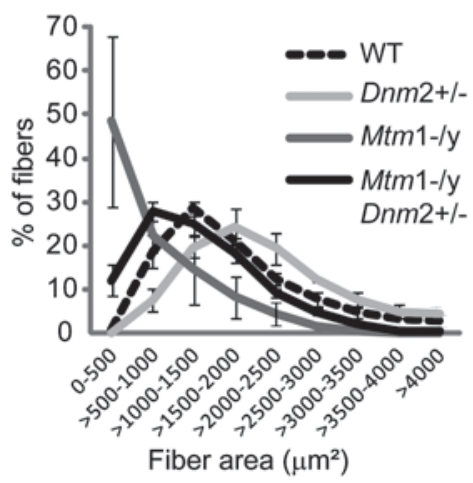

Dnm2+/-

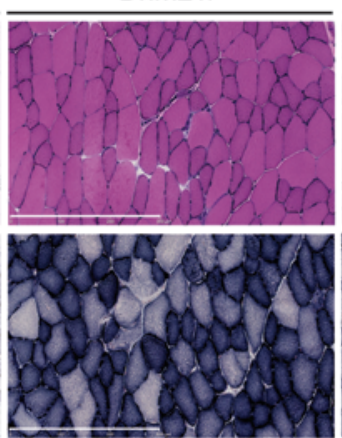

WT

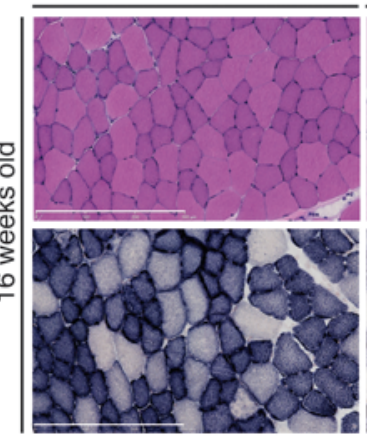

E

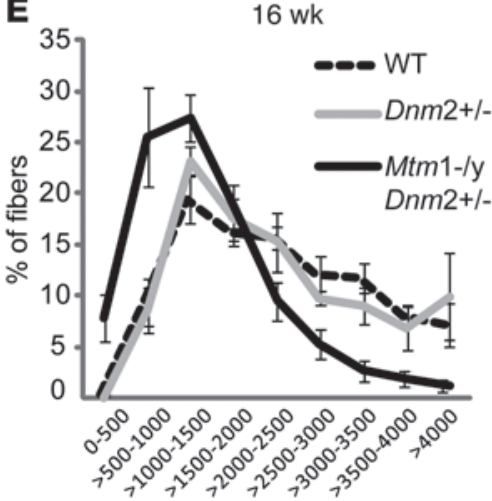

Fiber area $\left(\mu \mathrm{m}^{2}\right)$

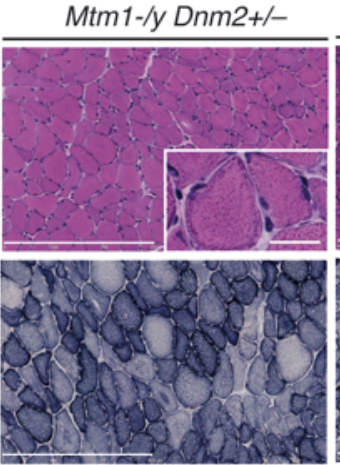

Dnm2+/-
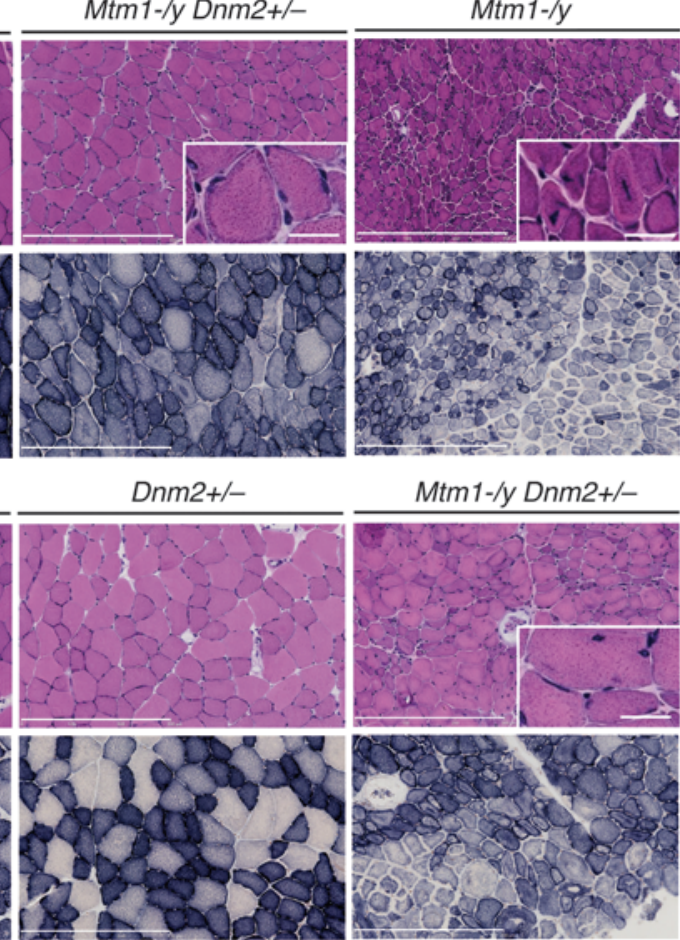

Mtm1-/y Dnm2+/-

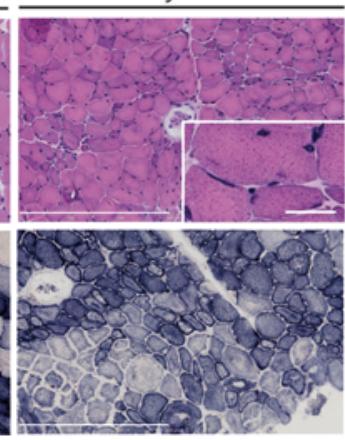

$\mathbf{F}$

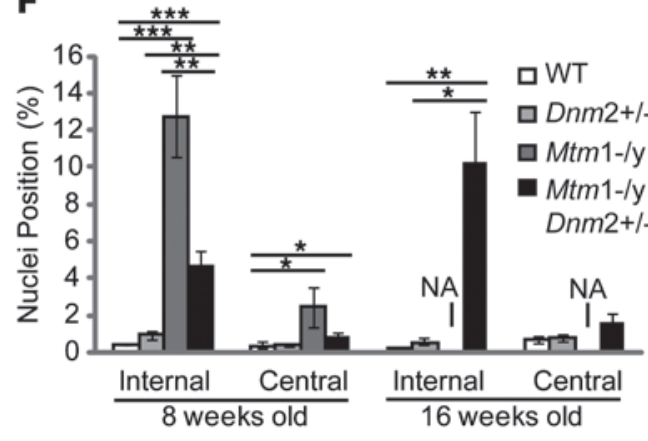

\section{Figure 3}

CNM histological features are greatly rescued in $M t m 1^{-1 y}$ mice with reduced DNM2 expression. Transverse TA sections from 8-week-old (A) or 16-week-old (C) mice were stained with H\&E (upper panel) or SDH (lower panel). Scale bars: $300 \mu \mathrm{m} ; 25 \mu \mathrm{m}$ (high magnification). (B) Transverse muscle sections from 8-week-old mice viewed by TEM. Arrow indicates membrane accumulation around nucleus. Scale bar: $0.5 \mu \mathrm{m}$. Transverse TA muscle sections from 8-week-old mice (D) and 16-week-old (E) mice were analyzed for fiber area. Fiber size is grouped into 500 - $\mu \mathrm{m}^{2}$ intervals, and represented as the percentage of total fibers. $n=5-7$ mice. $(F)$ The frequency of fibers with internal or central nuclei was scored. $n=5$ mice. Internal nuclei are defined as not subsarcolemmal or central. Images were not analyzed in $M$ tm $^{-1 / y}$ mice at 16 weeks, as they usually die before this age. All graphs depict mean \pm SEM. ${ }^{\star} P<0.05 ;{ }^{* \star} P<0.01 ;{ }^{* \star *} P<0.001$.

$M t m 1^{-/ y} \mathrm{Dnm} 2^{+/-}$mice compared with WT mice, as expected, at both 8 weeks and 6 months of age (Figure 2C). Mtm $1^{-/ y}$ mice exhibited an increase in DNM2 levels in diaphragm compared with WT mice at 8 weeks, consistent with results from 5 -week-old mice (Figure 1F). In the gastrocnemius (Figure 2D), TA (Figure $2 \mathrm{E}$ ), and soleus (Figure $2 \mathrm{~F}$ ) muscles, the same trend was seen at 8 weeks, 16 weeks, and 6 months (Supplemental Figure 5). Therefore, DNM2 levels are consistently increased in $M t m 1^{-/ y}$ mice and

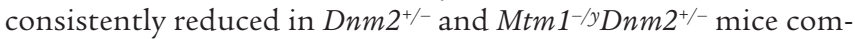
pared with WT mice in different muscles at different ages.
To determine whether the varied DNM2 protein level is due to altered protein synthesis, we performed quantitative RT-PCR (qRT-PCR) analysis on 8-week-old TA muscle lysates. The mRNA Dnm2 levels in both $\mathrm{Dnm} 2^{+/-}$and $M t m 1^{-/ y} \mathrm{Dnm} 2^{+/-}$mice were significantly reduced compared with WT and $M t m 1^{-1 y}$ mice (Figure $2 \mathrm{G}$ ), correlating with DNM2 protein expression (Figure 2E). No significant increase was seen in Dnm2 mRNA expression in $\mathrm{Mtm} 1^{-/ y}$ muscles, indicating that increased DNM2 protein expression in $M t m 1^{-1 y}$ muscles may be due to increased stabilization of DNM2 or reduced degradation rather than increased transcription. 
A
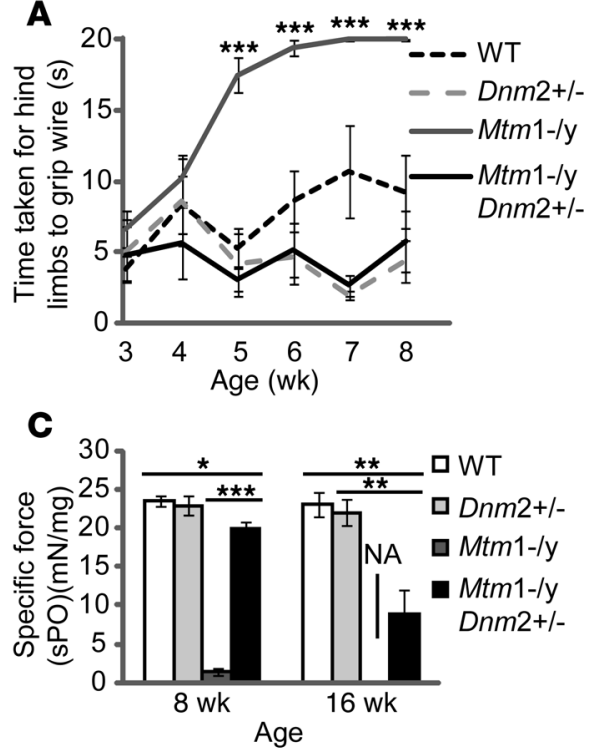

B
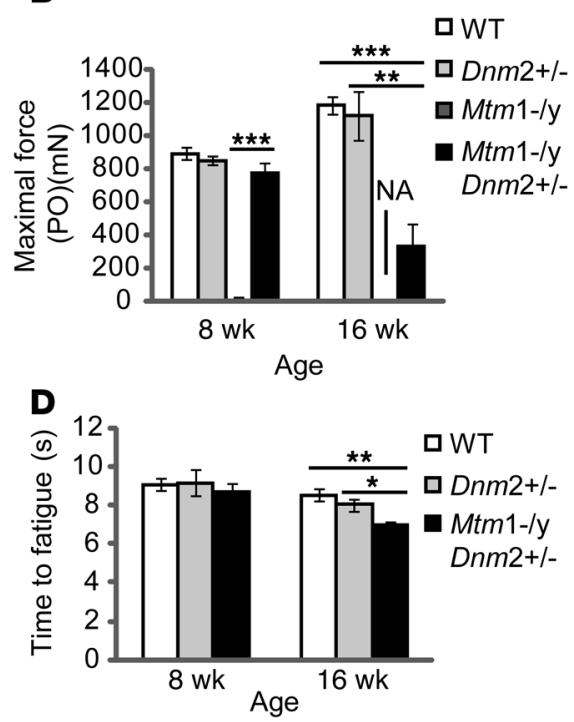

Figure 4

Improved muscle strength and endurance of $\mathrm{Mtm}^{-/ y}$ mice with reduced DNM2 expression. (A) The string test was performed on mice weekly from 3 to 8 weeks. A fall was considered equal to 20 seconds. (B) The absolute maximal force of the TA muscle was measured in 8-week-old and 16-week-old mice. (C) Specific maximal force of the TA. Mtm $1^{-1 / y}$ mice usually die before 16 weeks and were therefore not measured at that age. (D) Fatigue in TA muscle, measured as the time taken to reach $50 \%$ of maximum muscle force. Muscle fatigue was unable to be measured in $M t^{-1 / y} 1^{-l y}$ mice at 8 weeks due to extreme muscle weakness. All graphs depict mean \pm SEM. ${ }^{*} P<0.05$; ${ }^{* *} P<0.01 ;{ }^{* \star \star} P<0.001 . n=$ minimum 5 mice per group.
As the TA was one of the most affected muscles in $M t m 1^{-/ y}$ mice $(10,15)$, we investigated the localization of MTM1 and DNM2 by immunofluorescence analysis in TA from 8-week-old mice. The $Z$-line, identified by $\alpha$-actinin staining, appeared relatively undisturbed (Supplemental Figure 3A). DNM2 colocalized with $\alpha$-actinin at the $Z$-line and appeared relatively unperturbed in $M t m 1^{-/ y}$ and $M t m 1^{-/ y} D n m 2^{+/-}$mice (Supplemental Figure 3A). MTM1 was barely detectable in $M t m 1^{-/ y}$ and $M t m 1^{-/ y} \mathrm{Dnm} 2^{+/-}$mice, as expected (Supplemental Figure 3B). These data show that reducing expression of DNM2 rescues the life span and body weight of Mtm 1- $1^{-y}$ mice to WT level.

The 3' UTR region of DNM2 contains a site for miR-133a binding, resulting in repression of protein expression, and lack of miR133a was shown to cause CNM-like features in mice, corresponding with an increase in DNM2 expression (30). qRT-PCR analysis performed on 8-week-old TA lysates from $M t m 1^{-1 / y}$ mice identified no significant difference in miR-133a expression (Supplemental Figure 4A), despite the increase in DNM2 level (Figure 2E). miR-133awas, however, significantlyincreased in $\mathrm{Dnm} \mathrm{2}^{+/-}$and $\mathrm{Mtm}^{-/ y}$ $D n m 2^{+/-}$mice (Supplemental Figure 4). The 1.5 -fold increase in miR-133a, however, did not lead to a stronger decrease in DNM2 level than expected in $\mathrm{Dnm} 2^{+/-}$mice (50\%, Figure 2E). The molecular mechanisms causing increased miR-133a expression are unclear at present.

Muscle atrophy in $\mathrm{Mtm}^{-1}$ y mice is rescued by reducing DNM2 expression. To analyze further the effect of reducing DNM2 expression in $M t m 1^{-1 y}$ mice, we measured muscle mass. The fast-twitch gastrocnemius muscle was atrophied in $M t m 1^{-/ y}$ mice; however, no atrophy was observed in $M t m 1^{-/ y} \mathrm{Dnm} 2^{+/-}$mice analyzed at up to 1 year of age (Figure $2 \mathrm{H}$ ). Likewise, other fast-twitch muscles, the extensor digitorum longus (EDL) and plantaris muscle, did not exhibit atrophy in $\mathrm{Mtm}^{-/ y} \mathrm{Dnm} 2^{+/-}$mice at up to 1 year of age compared with WT mice (Supplemental Figure 4, B and C). The TA showed a strong atrophy in $\mathrm{Mtm}^{-/ y}$ mice compared with $\mathrm{Mtm}^{-/ y} \mathrm{Dnm} 2^{+/-}$, WT, and $D n m 2^{+/-}$mice at 8 weeks (Figure 2I). $M t m 1^{-/ y} D n m 2^{+/-}$TA weights were indistinguishable from those of WT mice at this age. At 16 weeks, unlike $M t m 1^{-/ y}$ mice, $M t m 1^{-/ y} D n m 2^{+/-}$mice were still alive (Figure $2 \mathrm{~A}$ ) and presented with some TA atrophy compared with WT and $\mathrm{Dnm} 2^{+/-}$mice, indicating that TA atrophy in $\mathrm{Mtm} 1^{-/ y}$ $D n m 2^{+/-}$is delayed. Interestingly the slow-twitch soleus muscle exhibited atrophy in $\mathrm{Mtm}^{-1 /}$ mice at 8 weeks, whereas there was no atrophy in $\mathrm{Mtm}^{-/ y} \mathrm{Dnm} 2^{+/-}$mice at up to 1 year of age compared with WT mice (Figure 2J). Similar results were seen when whole muscle mass was measured relative to body weight (Supplemental Figure 4, D-F). No difference was observed in liver or heart weights between $\mathrm{Mtm}^{-/ y} \mathrm{Dnm} 2^{+/-}$and WT mice (Supplemental Figure 4, G and $\mathrm{H})$. Therefore, muscle atrophy was fully rescued in gastrocnemius and soleus muscles and strongly delayed in TA muscle following the reduction of DNM2 expression in $\mathrm{Mtm}^{-1 / y}$ mice.

CNM bistology is greatly rescued in $M t m 1^{-1} y$ mice by reducing DNM2 expression. CNM presents histologically with mislocalized internal nuclei and muscle fiber hypotrophy. We analyzed 2 main time points: early (8 weeks), when the majority of the $M t m 1^{-/ y}$ mice were still alive, and late (16 weeks), when $95 \%$ of $\mathrm{Mtm}^{-/ y}$ mice have died. At 8 weeks, Mtm $1^{-/ y}$ TA muscle exhibited characteristic mislocalization of nuclei (Figure 3, A and F), reduced fiber size (Figure 3, A and D), and abnormal succinate dehydrogenase (SDH) staining with subsarcolemmal and central accumulations (Figure 3A). Mtm1 1-y Dnm2 $2^{+/-}$TA muscles were histologically similar to those of WT and Dnm2 $2^{+/-}$mice, as observed for the gastrocnemius and soleus muscles (Supplemental Figure 4, I and J), with only a few abnormal fibers on SDH staining (Figure 3A). Fiber hypotrophy was rescued and internal and central nuclei significantly reduced compared with $M t m 1^{-/ y}$ mice (Figure 3, A, D, and F). In addition, membrane accumulations around the nucleus were reduced (Figure 3B). By 16 weeks, the TA phenotype from $M t m 1^{-/ y} \mathrm{Dnm} 2^{+/-}$mice was mixed, as some areas appeared healthy and other areas resembled 8-week-old $\mathrm{Mtm}^{-1 / y}$ muscle, with muscle fiber hypotrophy, mislocalization of nuclei, and abnormal SDH staining (Figure 3, C, E, and F). The localization of desmin, an MTM1-binding partner shown to be disrupted in $M t m 1^{-/ y}$ mice (33), was barely altered in $M t m 1^{-/ y} \mathrm{Dnm} 2^{+/-}$mice (Supplemental Figure $6 \mathrm{~A}$ ), indicating that normal desmin localization is restored in $\mathrm{Mtm}^{-/ y} \mathrm{Dnm} 2^{+/-}$mice. Overall, these results indicate the CNM phenotype in different muscles in $\mathrm{Mtm1}^{-/ y}$ mice is rescued or strongly delayed by reducing DNM2 expression. 


\section{A}
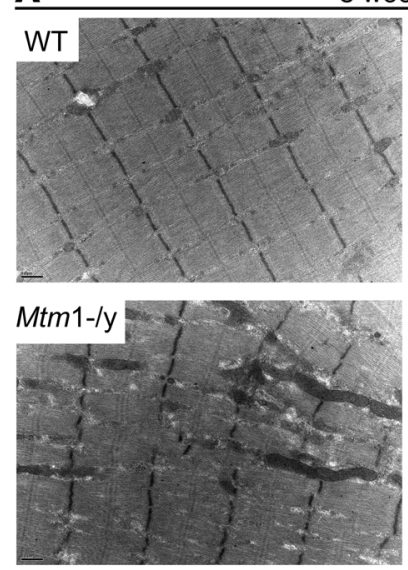

B
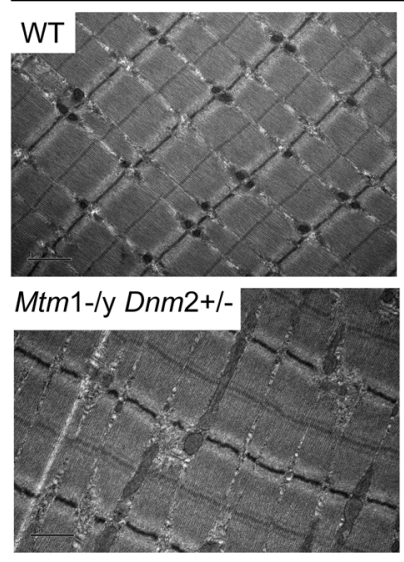

Figure 5

Improved muscle ultrastructure of $M t m 1^{-1 y}$ mice with reduced DNM2 expression. TA muscles from 8-week-old (A) and 16-week-old (B) mice were imaged by TEM. Scale bars: $0.5 \mu \mathrm{m}(\mathbf{A}) ; 1 \mu \mathrm{m}$ (B).

Reducing DNM2 expression improves muscle strength and performance of $\mathrm{Mtm}^{-1 y}$ mice. We next determined whether reducing DNM2 expression rescues the functional phenotype of $\mathrm{Mtm}^{-/ y}$ mice. The string test requires mice that are suspended by their front paws to lift and hold their hind limbs on the wire. While $M t m 1^{-/ y}$ mice fell off on several trials and were unable to perform the test by 8 weeks, $M t m 1^{-/ y} D n m 2^{+/-}$mice performed the test similarly to WT and Dnm2 $2^{+/-}$mice (Figure 4A and Supplemental Videos 2-4), indicating a rescue of whole-body strength. At 8 weeks, TA muscles from $M t m 1^{-1 y}$ mice exhibited extremely weak absolute and specific maximal muscle force, whereas $M t m 1^{-/ y} \mathrm{Dnm} 2^{+/-}$mice performed the test similarly to WT and $D n m 2^{+/-}$mice (Figure 4, B and C). At 16 weeks, the maximal force of the TA muscle of $M t m 1^{-/ y} \mathrm{Dnm} 2^{+/-}$mice was reduced, consistent with histological data. Furthermore, no change in fatigability was observed at 8 weeks; however, at 16 weeks, Mtm1-/y Dnm2 $2^{+-}$TA muscles fatigued faster than controls (Figure 4D). Notably histologically and physiologically, 16-week-old $\mathrm{Mtm} 1^{-/ y}$ $\mathrm{Dnm} \mathrm{2}^{+/-}$mice performed better than $\mathrm{Mtm} 1^{-/ y}$ mice at 8 weeks, indicating either a slower progression of the disease or rescue in some but not all muscle fibers, as indicated by the mixed phenotype observed histologically in the TA at 16 weeks (Figure 3C).
Overall, atrophy and decreased muscle force of the TA muscle in $M t m 1^{-/ y} \mathrm{Dnm} 2^{+/-}$mice is reduced and strongly delayed compared with that of $M t m 1^{-1 y}$ mice.

Improved muscle ultrastructure in $\mathrm{Mtm} 1^{-/ y} \mathrm{Dnm} 2^{+/-}$mice. We next analyzed the ultrastructure of $M t m 1^{-/ y} \mathrm{Dnm} 2^{+/-}$muscle by transmission electron microscopy (TEM). Eight-week-old $M t m 1^{-/ y} \mathrm{Dnm} 2^{+/-}$ TA morphology resembled that of WT and $D n m 2^{+/-}$muscles, with aligned $Z$-lines and sarcomeres and no obvious mitochondrial structural abnormalities, whereas $M t m 1^{-/ y}$ muscle displayed abnormal mitochondria shape, membrane accumulations, Z-line misalignment, and altered myofibrillar width (Figure 5A), as reported previously $(10,15,34)$. Notably, muscle from $M t m 1^{-/ y} D n m 2^{+/-}$mice at 16 weeks was heterogeneous; some regions appeared healthy, while other areas appeared disturbed (Figure 5B). Furthermore, mitochondrial abnormalities that were not evident at 8 weeks were detectable in some regions of 16 -week-old $M t m 1^{-/ y} \mathrm{Dnm} 2^{+/-}$mice. This supports our previous histological and physiological results, indicating that the CNM phenotype in $\mathrm{Mtm}^{-/ y} \mathrm{Dnm} 2^{+/-} \mathrm{TA}$ muscle is partially albeit substantially rescued at different time points.

Triad structures are normalized in $\mathrm{Mtm} 1^{-/ y} \mathrm{Dnm} 2^{+/-}$mice. A common feature shared between CNM patients and animal models of CNM is a disruption of the structure and position of triads within skeletal muscle $(13-15,35)$. DHPR $\alpha$, a voltage-dependent calcium channel found on T-tubules of mature muscles, was localized in punctuate structures within TA myofibers of WT, $D n m 2^{+/-}$, and $M t m 1^{-1 y} \mathrm{Dnm} 2^{+/-}$mice, consistent with normal T-tubule localization (Supplemental Figure 6B). However, in $M t m 1^{-1 y}$ muscle, this specific staining was lost, indicating severe disruption of the T-tubules. This was confirmed by staining for ryanodine receptor (RyR1), a calcium channel localized specifically at the sarcoplasmic reticulum of triads. Transverse and longitudinal images showed a rescue of RyR1 localization in most fibers from $M t m 1^{-/ y}$ $D n m 2^{+/-}$mice, with only a few fibers exhibiting RyR1 accumulations or perturbed localization, as seen extensively in $\mathrm{Mtm} 1^{-/ y}$ mice (Figure 6A). High-magnification TEM images confirmed strong disruption of T-tubule/triad structures in $M t m 1^{-/ y}$ mice compared with WT and $D n m 2^{+/-}$mice, whereas well-positioned triads were clearly visible in $\mathrm{Mtm}^{-/ y} \mathrm{Dnm} 2^{+/-}$mice (Figure 6B). Analysis of the triads confirmed no difference in the number of triads per sarcomere in WT, $\mathrm{Dnm} 2^{+/-}$, or $\mathrm{Mtm} 1^{-/ y} \mathrm{Dnm} 2^{+/-}$mice, whereas $\mathrm{Mtm} 1^{-/ y}$ mice exhibited a reduced number of triads per sarcomere (Figure 6C), as shown previously (34). Caveolin 3 is found at T-tubules during muscle development and regeneration and at the sarcolemma in mature muscle (36). While WT and Dnm2 $2^{+/-}$muscle showed caveolin 3 localizing to the sarcolemma as expected, many fibers from $M t m 1^{-1 y}$ mice exhibited a strong internal staining pattern of caveolin 3 (Figure 6D). This phenotype was largely rescued in $M t m 1^{-/ y} \mathrm{Dnm} 2^{+/-}$muscle, with only occasional fibers showing internal localization of caveolin 3. Therefore, the localization and structure of triads was rescued in 8-week-old $M t m 1^{-/ y} \mathrm{Dnm} 2^{+/-}$mice.

Long-term physiological phenotype of $\mathrm{Mtm}^{-/ y} \mathrm{Dnm} 2^{+/-}$mice. XLCNM presents with very severe muscle weakness in patients and lethality in $M t m 1^{-/ y}$ mice within 1 to 3 months; however, reducing DNM2 expression in these mice rescued the life span and greatly improved muscle strength at 8 and 16 weeks. We determined the phenotype of $\mathrm{Mtm}^{-/ y} \mathrm{Dnm} 2^{+/-}$mice at later time points to assess the extent of muscle function compatible with a normal life span. Mtm 1-y Dnm2 $2^{+/-}$mice aged 6 months and 12 months were able to move and perform basic tasks (Supplemental Video 5). Aged $M t m 1^{-/ y} \mathrm{Dnm}^{+/-}$mice looked similar to WT mice (Figure 
A
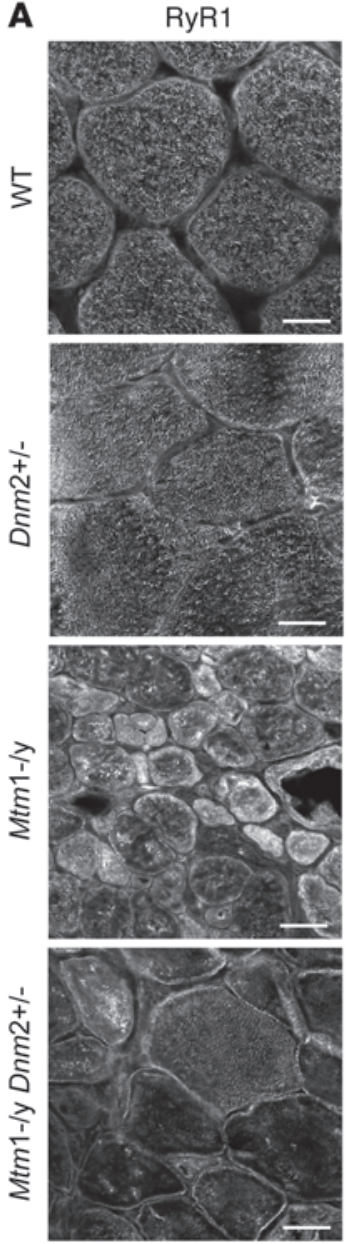

B

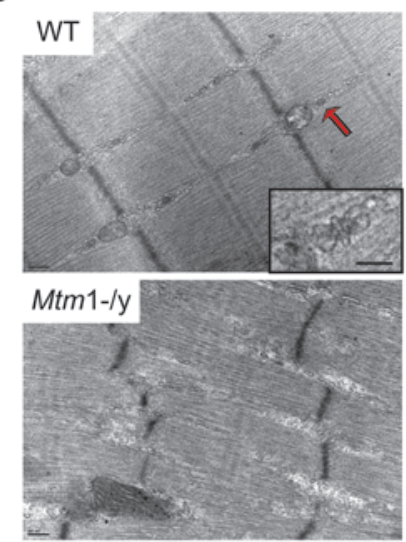

RyR1
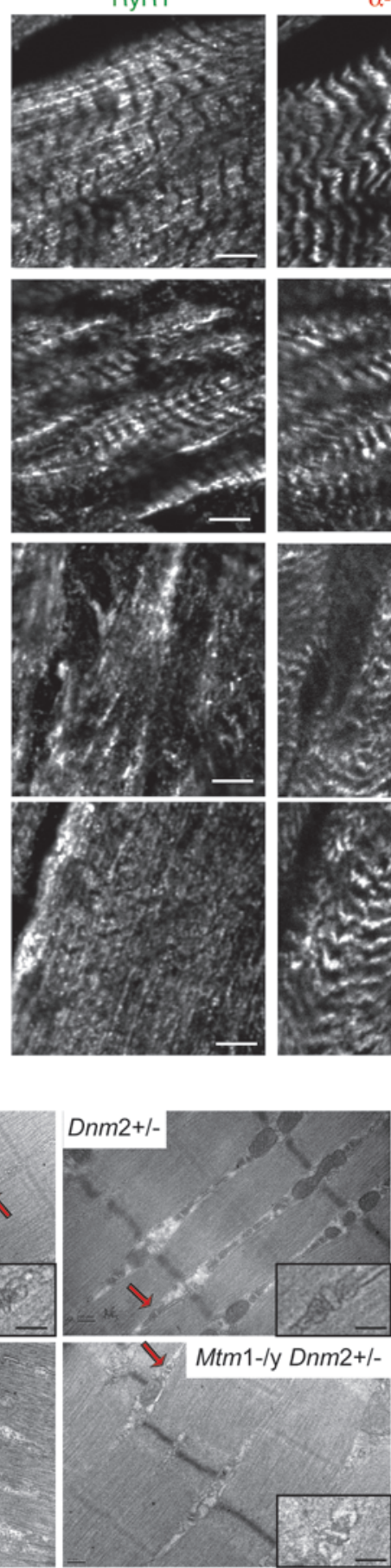

$\alpha$-actinin
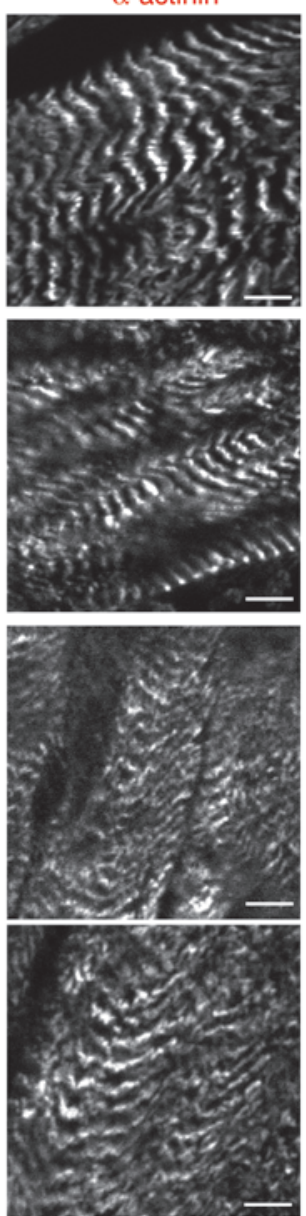

C
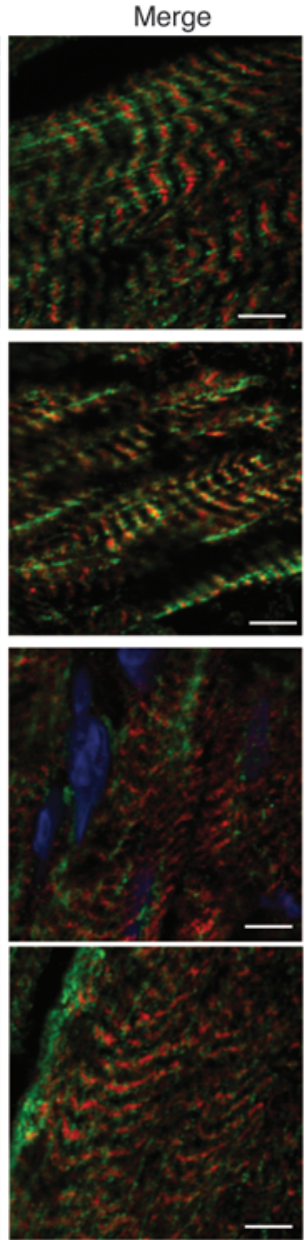

D
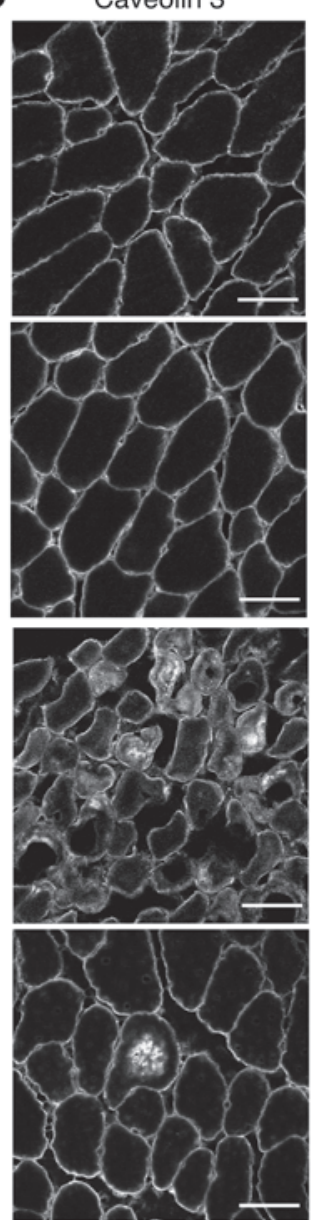

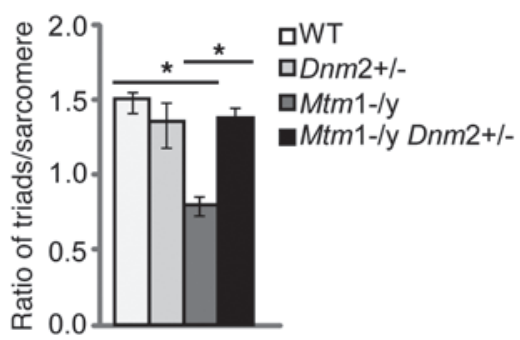

\section{Figure 6}

Integrity of triads in TA muscle from 8-week-old mice. (A) Transverse and longitudinal muscle sections were stained with RyR1 or costained with RyR1 (green) and $\alpha$-actinin (red) antibodies and imaged by confocal microscopy. Scale bars: $20 \mu \mathrm{m}$ (transverse); $5 \mu \mathrm{m}$ (Iongitudinal). (B) Muscles were imaged by TEM. Arrows point to normally localized triads, shown in high magnification insert. Scale bars: $200 \mathrm{~nm} ; 100 \mathrm{~nm}$ (high magnification). (C) Percentage of triads visualized per sarcomere. Graph depicts mean \pm SEM. ${ }^{*} P<0.05$. (D) Transverse muscle sections were stained with caveolin 3 and imaged by confocal microscopy. Scale bars: $50 \mu \mathrm{m}$.

7A), but walked with hind feet pointing outwards (Figure 7B and Supplemental Figure 7A). This feature was not progressive from 6 to 12 months. To determine overall maximal leg strength, the grip-strength test was performed using a dynamometer. No dif- ference in strength of the 2 front paws was observed between WT and $\mathrm{Mtm} 1^{-/ y} \mathrm{Dnm} 2^{+/-}$mice during the first 12 months (Supplemental Figure 7B). A small reduction in strength was observed in $M t m 1^{-y} \mathrm{Dnm} 2^{+/-}$mice compared with WT mice when all 4 
A
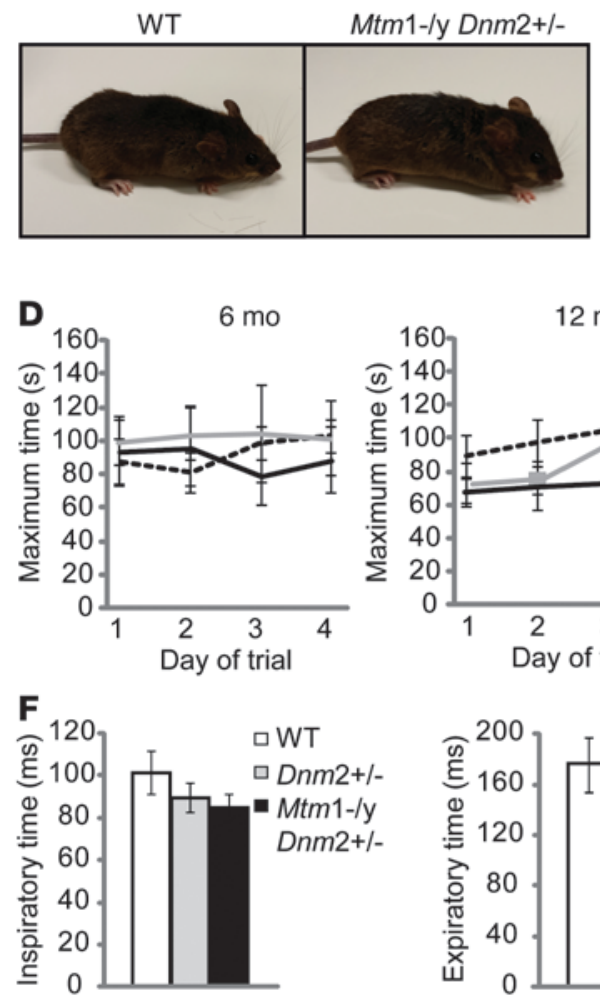

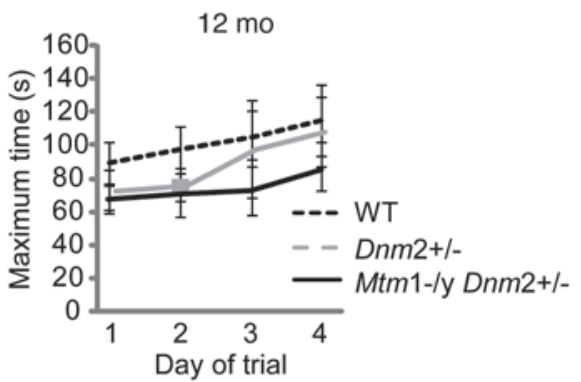

B

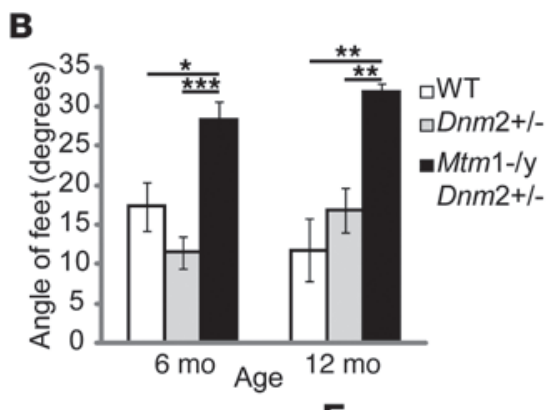

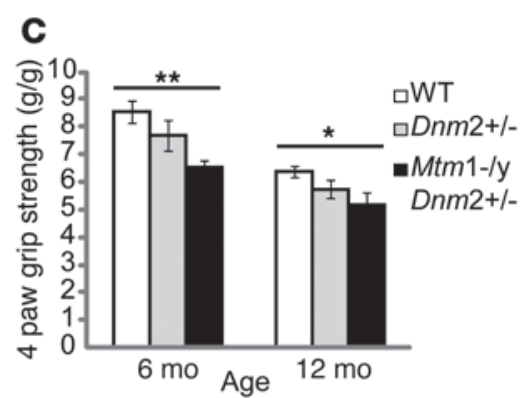

E

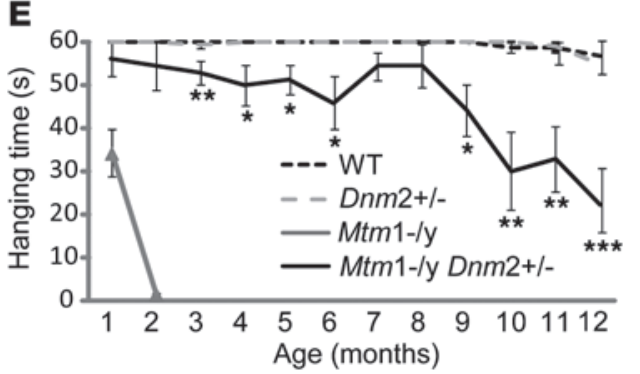

G
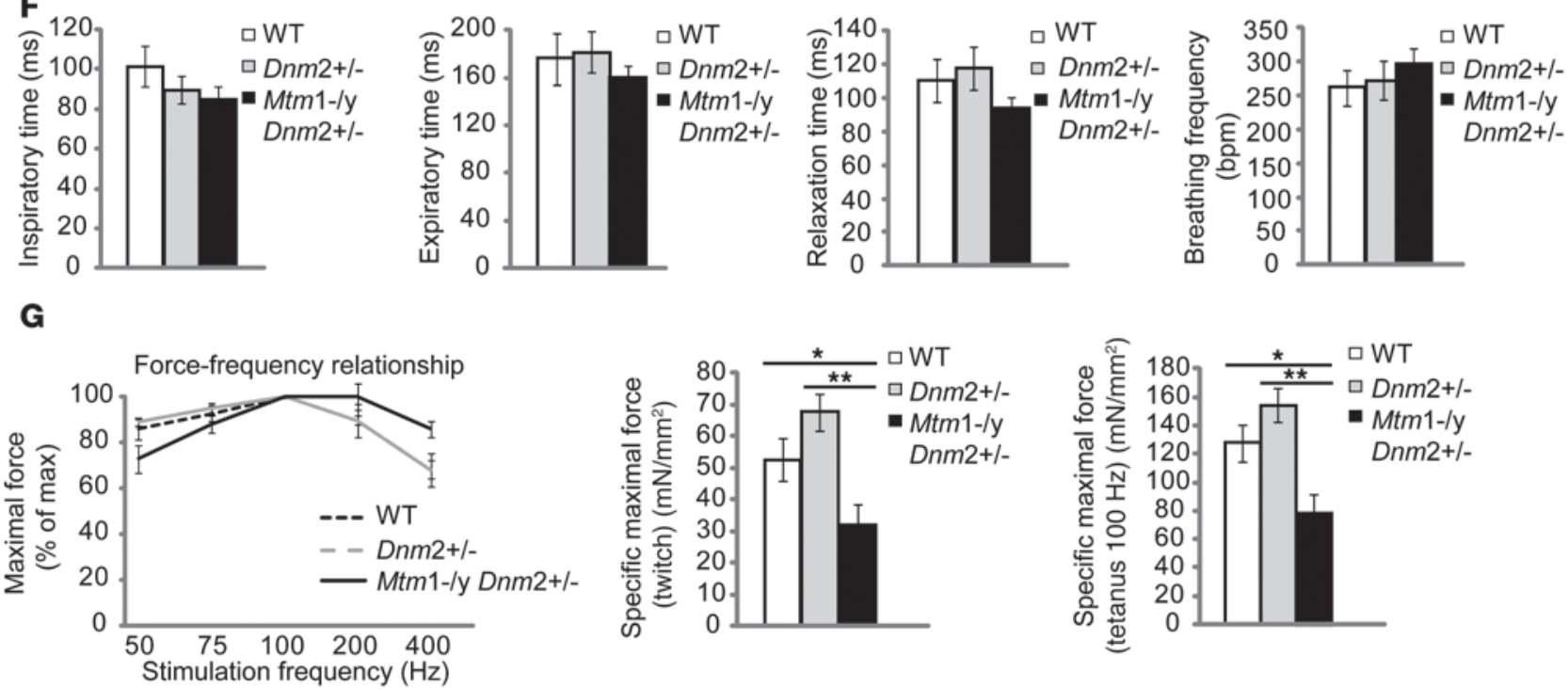

H
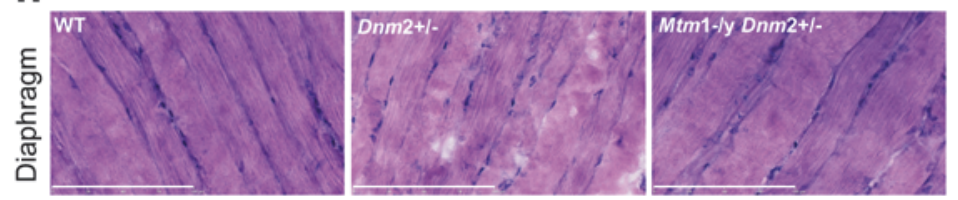

Figure 7

Long-term phenotype of Mtm1-/y mice with reduced DNM2 expression. (A) 12-month-old WT (left) and Mtm1-ly Dnm2 ${ }^{+/-}$(right) mice. (B) Footprint test indicating the angle of hind foot position. Raw data in Supplemental Figure 7. (C) Four-paw grip test. (D) Rotarod test performed under acceleration mode (4-40 rpm in 5 minutes). $n=3$ trials/mouse/d. (E) The hanging test requires mice to be suspended from a cage lid for up to 60 seconds. $n=3$ trials/mouse (F) A plethysmograph test for resting breathing measurements was performed on 6-month-old mice. Inspiratory/expiratory/relaxation time, and breathing frequency are shown; complementary results in Supplemental Figure 8. (G) Maximal muscle force was measured in diaphragm from 6-month-old mice. Force-frequency relationship and specific maximal force under twitch and tetanus (100 Hz) are depicted. (H) Longitudinal diaphragm sections were stained with H\&E. Scale bars: $100 \mu \mathrm{m}$. All graphs depict mean $\pm \mathrm{SEM} .{ }^{*} P<0.05 ;{ }^{* \star} P<0.01 ;{ }^{* \star} P<0.001 . n=$ minimum 5 mice.

paws were measured, at both 6 months and 12 months (Figure 7C), indicating the hind limbs of $M t m 1^{-/ y} \mathrm{Dnm} 2^{+/-}$mice exhibit reduced maximal muscle force compared with WT mice, which was not progressive. The rotarod test for general motor coor- dination, strength, and endurance was performed, with no difference observed at 6 or 12 months (Figure 7D), confirming the general coordination and overall strength of $\mathrm{Mtm} \mathrm{1}^{-/ y}$ $D n m 2^{+/-}$mice was not severely perturbed. The hanging test is a 


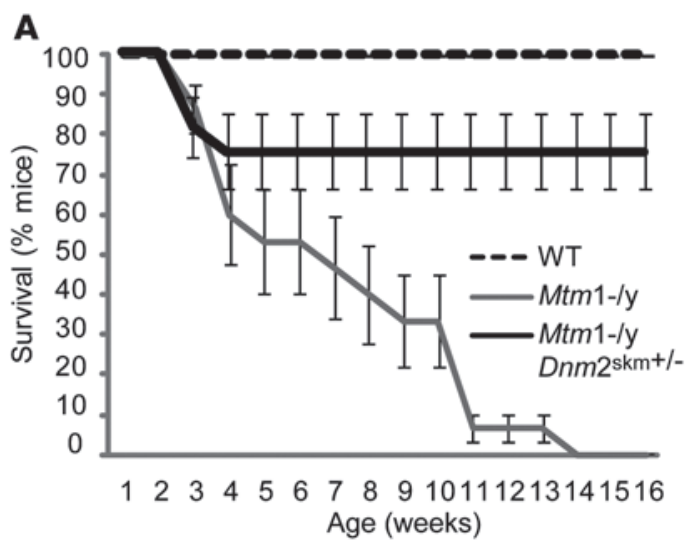

C
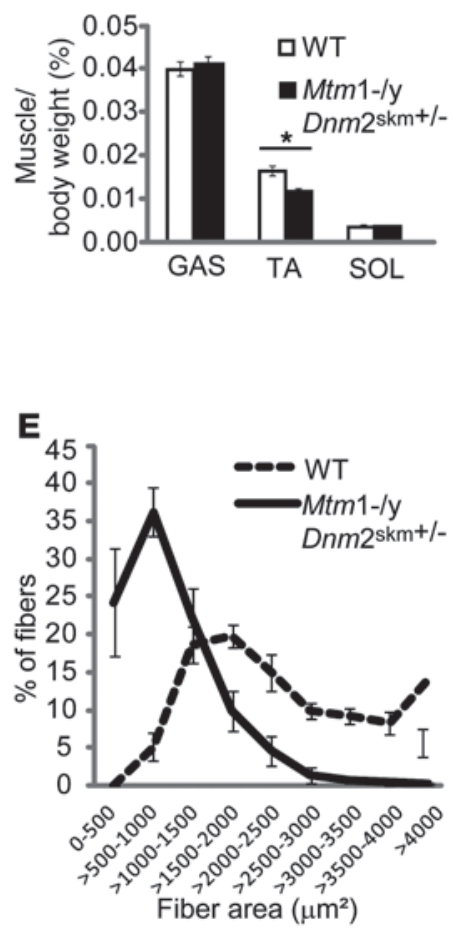

D
B

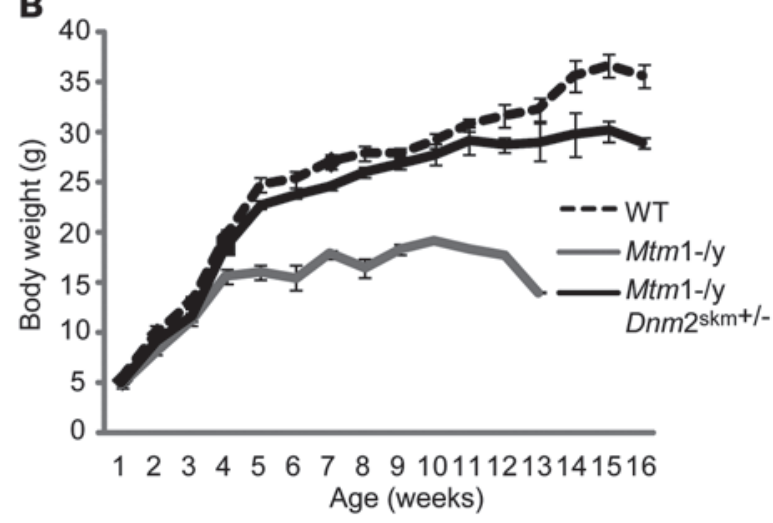

TA

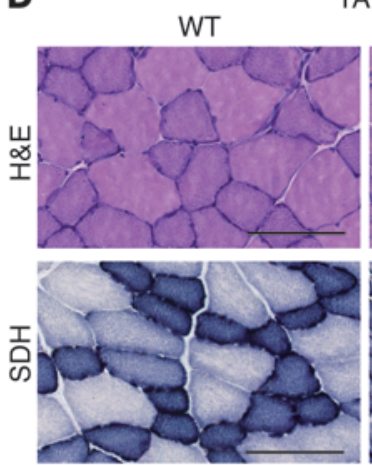

G
$\mathbf{F}$

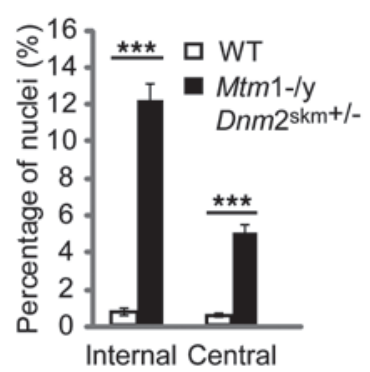

Mtm1-/y Dnm2 $2^{\text {skm+/- }}$
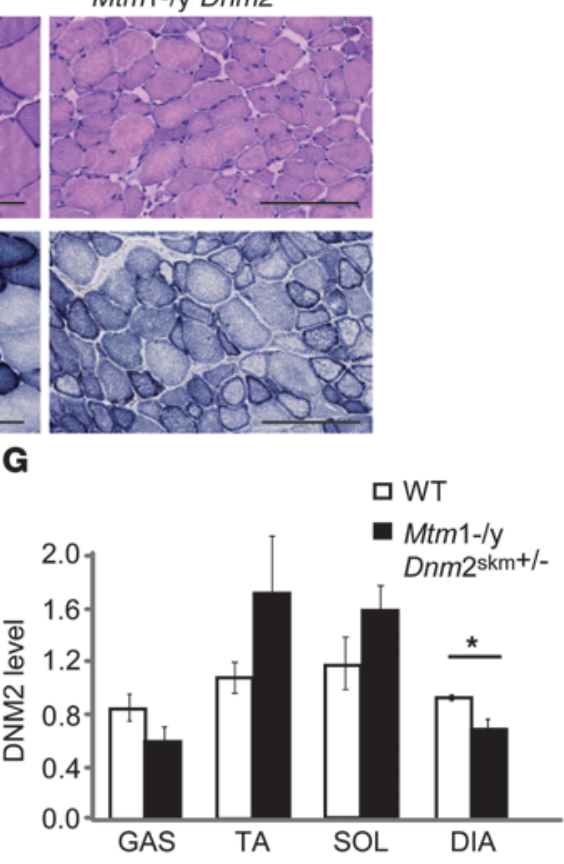

\section{Figure 8}

Reducing DNM2 in skeletal muscle alone ameliorates the life span and pathology of $M t m 1^{-1 / y}$ mice. (A) Life span of all mice represented as a

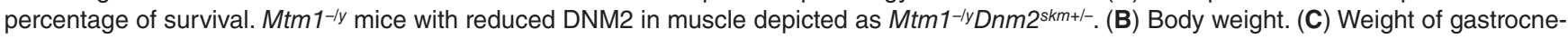
mius, TA, and soleus muscles as a percentage of total body weight. (D) Transverse TA sections were stained with H\&E (top panel) or SDH (lower panel). Scale bars: $100 \mu \mathrm{m}$. (E) Transverse sections were analyzed for fiber area. Fiber size is grouped into $500 \mu \mathrm{m}^{2}$ intervals, and represented as a percentage of total fibers. $(\mathbf{F})$ The frequency of fibers with internal or central nuclei was counted in TA muscle. (G) Relative level of DNM2 protein from TA muscles, standardized to GAPDH. DNM2 level is represented as a fold difference from WT lysate. All graphs depict mean + SEM. ${ }^{*} P<0.05 ;{ }^{* * *} P<0.001 . n=4-12$ mice, aged 16 weeks.

strenuous test that requires mice to be suspended from a cage lid for 60 seconds. The severely affected $M t m 1^{-1 y}$ mice were unable to perform this test after 1 month (Figure 7E). In comparison, $M t m 1^{-/ y}$ Dnm2 $2^{+/-}$mice could perform this test up to the last age tested (12 months), albeit to a lesser extent than WT mice (Figure 7E and Supplemental Videos 6-8). As $M t m 1^{-/ y} \mathrm{Dnm} 2^{+/-}$mice were able to successfully perform basic motor strength tests at up to 12 months of age, we conclude that the disease progression was stopped over time and life span and basic motor function were rescued.
Normal long-term diaphragm function in $\mathrm{Mtm}^{-/ y} \mathrm{Dnm} 2^{+/-}$mice. We have shown that individual muscles appear to be differently affected in $M t m 1^{-1 y}$ mice, and the corresponding rescue by reduction of DNM2 is varied between muscles (Figure 2, C-J, and Supplemental Figure 4). The main concern for the longevity of patients with XLCNM is the diaphragm's function, as they have life-threatening respiratory failure (1). In addition, we found the histology of the diaphragm in 5-week-old $M t m 1^{-/ y}$ mice was strongly altered (Figure $1 \mathrm{G})$. We thus tested the function of the diaphragm in 6-month-old 
A

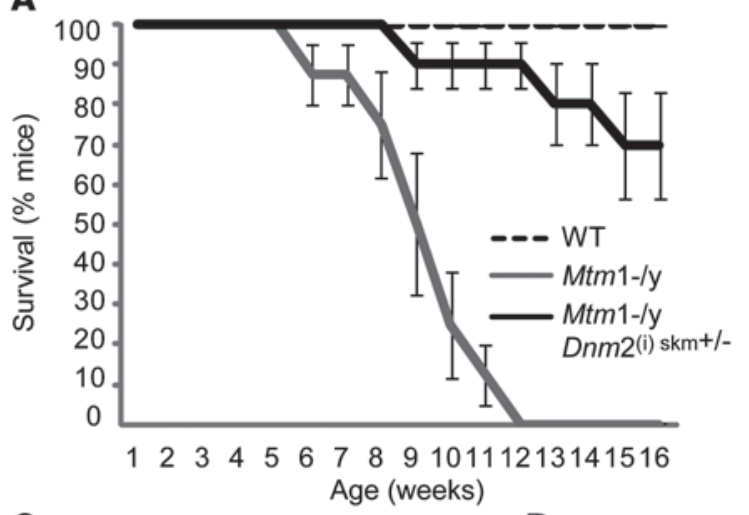

C

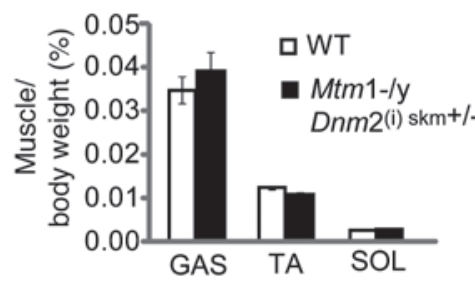

E

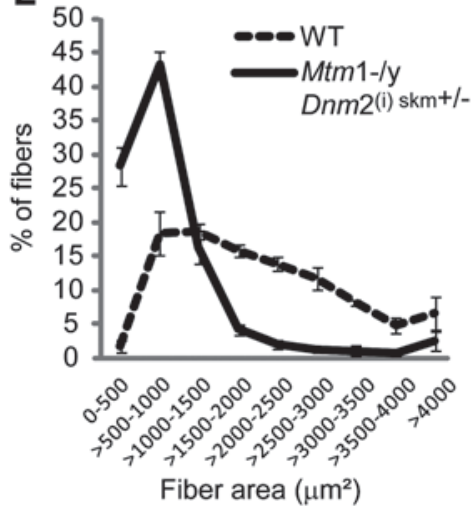

B

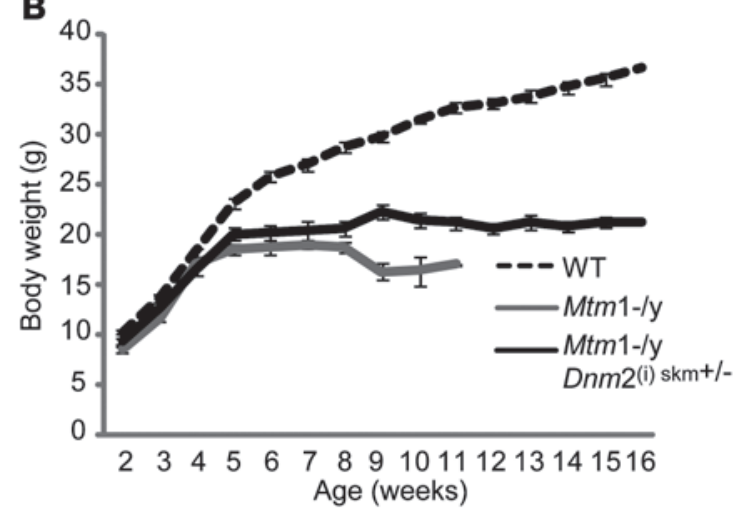

TA
WT

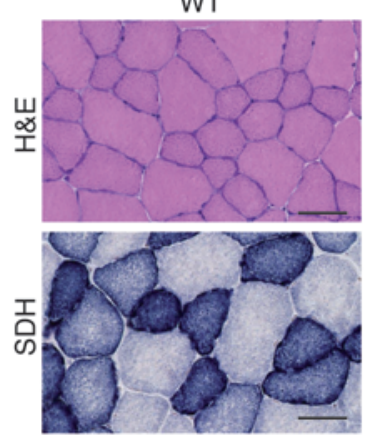

$\mathbf{F}$

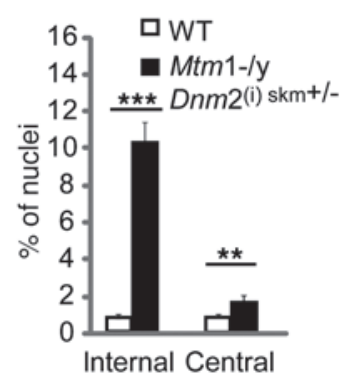

Mtm1-/y Dnm2 $2^{(0)}$ skm+/-

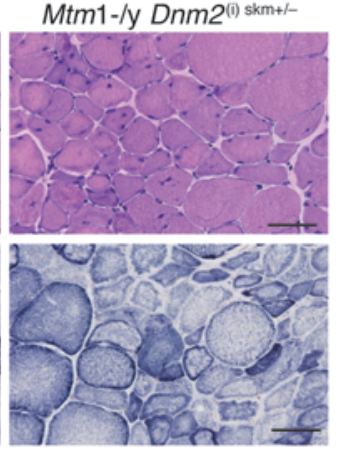

G

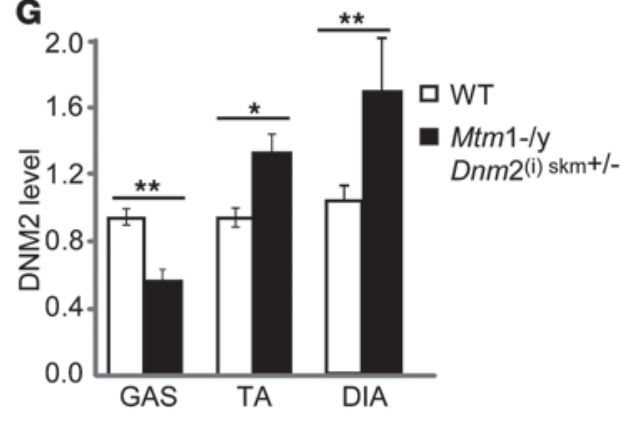

Figure 9

Reducing DNM2 in skeletal muscle after the onset of symptoms ameliorates the life span and pathology of $\mathrm{Mtm} 1^{-1 /}$ mice. (A) Life span of all mice represented as a percentage of survival. (B) Body weight. (C) Weight of gastrocnemius, TA, and soleus muscles represented as a percentage of total body weight. (D) Transverse TA sections were stained with H\&E (top panel) or SDH (lower panel). Scale bars: $100 \mu \mathrm{m}$. (E) Transverse sections were analyzed for fiber area. Fiber size is grouped into $500 \mu \mathrm{m}^{2}$ intervals. (F) The frequency of fibers with internal or central nuclei were counted. (G) Relative level of DNM2 protein from TA muscles, standardized to GAPDH. DNM2 level is represented as a fold difference from WT control lysate. All graphs depict mean + SEM. ${ }^{\star} P<0.05 ;{ }^{* \star} P<0.01 ;{ }^{* \star \star} P<0.001 . n=4-12$ mice, aged 16 weeks.

mice using the plethysmograph test to measure the spontaneous breathing pattern under resting conditions. Mtm $1^{-/ y} \mathrm{Dnm2^{+/- }}$ mice performed similarly to WT and $D n m 2^{+/-}$mice, with no significant difference detected (Figure 7F and Supplemental Figure 8). No significant difference in the force-frequency relationship was detected in isolated strips of diaphragm; however, a reduction in specific maximal force was observed (Figure $7 \mathrm{G}$ ). Histologically, Mtm1-/y $D n m 2^{+/-}$diaphragm resembled that of WT and $D n m 2^{+/-}$muscle, with no major alterations to nuclei positioning or fibrosis observed (Figure $7 \mathrm{H})$. The diaphragm of $M t m 1^{-/ y} \mathrm{Dnm} 2^{+/-}$mice sustained reduced
DNM2 protein levels at 6 months (Figure 2C), compared with $M t m 1^{-1 y}$ mice in which DNM2 expression was elevated at 5 weeks (Figure 1, E and F) and 8 weeks (Figure 2C). Overall, the diaphragm muscle of $\mathrm{Mtm}^{-/ y} \mathrm{Dnm} 2^{+/-}$mice was indistinguishable from that of control mice, supporting the extensive phenotypic amelioration of the XLCNM phenotypes upon DNM2 reduction.

Muscle-specific reduction of DNM2 is sufficient to rescue the phenotype and improve the life span in Mtm1-1y mice. In this study, we were able to fully rescue the life span of $\mathrm{Mtm}^{-/ y}$ mice and most of the clinical and histological features of the disease by reducing DNM2 expression in utero in all 
tissues. To determine whether the rescue of the muscle phenotype was cell-autonomous, we crossed HSA-Cre (where HSA indicates human skeletal muscle $\alpha$-actin) and HSA Cre-ER ${ }^{\mathrm{T} 2}$ mice (37) with floxed Dnm2 mice to produce $\mathrm{Dnm} 2^{\mathrm{skm+}+-}$ (Cre positive) and $\mathrm{Dnm} 2^{(i) \mathrm{skm+/}}\left(\mathrm{Cre}-\mathrm{ER}^{\mathrm{T} 2}\right.$ ) mice. These mice were then crossed with $M t m 1^{-/ y}$ mice to produce tissue-specific excision of Dnm2. When DNM2 expression was reduced in muscle ( $\mathrm{Mtm}^{-1 / y} \mathrm{Dnm} 2^{\mathrm{skm+}+-}$, HSA promoter active from $9 \mathrm{dpc}$; ref. 38), we were able to increase the life span of $\mathrm{Mtm}^{-/ y} \mathrm{Dnm} 2^{\mathrm{skm+} /-}$ mice, with $75 \%$ surviving until at least 16 weeks, while no $\mathrm{Mtm}^{-1 / y}$ mice survived to this age (Figure 8A), consistent with our results from $\mathrm{Mtm}^{-/ y} \mathrm{Dnm} 2^{+/-}$ mice (Figure 2A). Four WT and $4 \mathrm{Mtm} 1^{-/ y} \mathrm{Dnm} 2^{\mathrm{skm+} /-}$ mice have been kept alive for future long-term analysis, and all mice were 12 months old at the time of publication. A corresponding increase in body weight was also observed in $\mathrm{Mtm}^{-/ y} \mathrm{Dnm} 2^{\mathrm{skm+}+-}$ mice compared with $\mathrm{Mtm} 1^{-/ y}$ mice (Figure 8B). No difference in mass of the gastrocnemius or soleus muscles was observed between $\mathrm{Mtm} 1^{-/ y} \mathrm{Dnm} 2^{\mathrm{skm+}+-}$ and WT mice (Figure 8C). At 16 weeks, when all $\mathrm{Mtm}^{-/ y}$ littermates had died, the TA of $\mathrm{Mtm} 1^{-/ y} \mathrm{Dnm} 2^{\mathrm{skm+} /-}$ mice exhibited some atrophy, with a reduction in muscle mass and fiber size compared with WT littermates, associated with increased central and internal nuclei and some abnormal SDH staining (Figure 8, D-F), similar to that seen in the TA muscle from 16-week-old $\mathrm{Mtm}^{-/ y} \mathrm{Dnm} 2^{+/-}$mice (Figure 2). These alterations were less pronounced than those seen in $\mathrm{Mtm}^{-/ y}$ mice at 8 weeks. Importantly, the gastrocnemius and soleus of $\mathrm{Mtm}^{-/ y} \mathrm{Dnm} 2^{\mathrm{skm}+/-}$ mice did not exhibit a significant difference in fiber size or nuclei position compared with WT mice (Supplemental Figure 9, A-D), indicating the phenotype is rescued differently in different muscles. DNM2 protein levels were measured in different muscles at 16 weeks, and there was a significant reduction in DNM2 expression in the diaphragm, but not in other muscles measured, compared with WT (Figure 8G and Supplemental Figure 9, E-H). Importantly, $\mathrm{Mtm}^{-1 / y}$ mice were not alive at this age to compare the levels of DNM2, which were already increased in these muscles at younger ages (Figure 2). As the diaphragm is a vital muscle required for breathing, we propose that reduced DNM2 expression in the diaphragm muscle is critical for increased survival of $M t m 1^{-/ y} \mathrm{Dnm} 2^{\mathrm{skm+}+-}$ mice and to rescue XLCNM.

Muscle-specific reduction of DNM2 after birth is sufficient to rescue $M t m 1^{-1 y}$ mice. We crossed $M t m 1^{-/ y}$ mice with $D n m 2^{+/-}$mice under the HSA-Cre ER ${ }^{\mathrm{T} 2}$ system to allow excision of DNM2 after birth in muscle, induced by tamoxifen injection (37). Importantly, tamoxifen injections were performed when mice were 3 weeks old, after the onset of muscle atrophy (Figure 2) and centralized nuclei (15). We found that $70 \%$ of the injected $M t m 1^{-/ y} \mathrm{Dnm} 2^{(i) s k m+/-}$ mice survived to 16 weeks (Figure 9A). A higher body weight was observed compared with that of $M t m 1^{-/ y}$ mice; however at 16 weeks, body weight was still significantly reduced compared with that of WT mice (Figure 9B). No difference in normalized mass of the gastrocnemius, soleus, or TA muscles was observed compared with WT mice at 16 weeks (Figure 9C), unlike $M t m 1^{-1 y}$ mice at earlier time points (Figure 2). Further analysis of TA muscles from $M t m 1^{-/ y} \mathrm{Dnm} 2^{(i) s k m+/-}$ mice showed some fiber hypotrophy (Figure 9, D and E), associated with increased central and internal nuclei, and abnormal SDH staining (Figure 9, D and F), similar to the TA muscle from 16-week-old Mtm1 $1^{-/ y} \mathrm{Dnm} 2^{+/-}$(Figure 3). We noted a decrease in DNM2 protein expression in gastrocnemius from $M t m 1^{-/ y} \mathrm{Dnm} 2^{(i) s k m+/-}$ mice at 16 weeks compared with WT mice; however, an increase in DNM2 protein expression was observed in TA and diaphragm muscles (Figure 9G and Supplemental Figure 10). These differences may be due to differential efficiency in DNM2 excision upon tamoxifen-mediated activation of the Cre recombinase. The increased DNM2 expression in the diaphragm at 16 weeks may be correlated with the reduced survival rate in $M t m 1^{-/ y} \mathrm{Dnm} 2^{(i) s k m+/-}$ mice from 8 to 16 weeks. Therefore, we conclude that reduction of DNM2 levels in muscle after birth, after the onset of symptoms, is sufficient to improve the life span and the CNM phenotype observed in $M t m 1^{-/ y}$ mice.

\section{Discussion}

In this study we showed that reducing DNM2 in $M t m 1^{-/ y}$ mice rescues most features of XLCNM and greatly prolongs life span and long-term muscle and motor performance. Furthermore, reduction of DNM2 in muscle alone, and after the onset of symptoms, is sufficient to rescue the phenotype.

DNM2 and MTM1 function in a common pathway. Loss-of-function mutations in MTM1 and dominant mutations in DNM2 lead to CNM in human and CNM phenotypes in mice models $(1,9)$. Moreover, biochemical analysis suggests several DNM2 CNM mutants increase DNM2 GTPase activity and oligomerization $(26,27)$. Furthermore, overexpression of WT DNM2 by AAV or by miR-133a inhibition resulting in increased DNM2 expression promoted muscle defects and centralization of nuclei in mouse muscle $(28,30)$. We thus hypothesized DNM2-CNM mutations are gain-of-function. However, no link between MTM1 and DNM2 functions was previously established. As lowering the genetic dosage of DNM2, either systemically or in skeletal muscle, strongly ameliorated the XLCNM phenotype of $\mathrm{Mtm}^{-1}{ }^{-1}$ mice, we have identified MTM1 and DNM2 as being linked specifically in muscle, in a common pathway controlling muscle mass and maximal force. Moreover, we suggest MTM1 is a negative regulator of the DNM2 pathway in muscle, as decreased DNM2 can compensate for absence of MTM1.

These data represent a first link between MTM1 and DNM2 in the pathogenesis of CNM. Moreover, the rescue data presented here support that DNM2-CNM mutations are gain-of-function in skeletal muscle. We thus hypothesize that several forms of CNM are due to increased expression and/or function of DNM2. In particular, MTM1 loss in mice, and potentially in human, may lead to CNM phenotypes through overexpression and/or overactivation of DNM2.

Interestingly, we noted increased DNM2 protein expression in XLCNM patients and in $M t m 1^{-/ y}$ muscle (Figure 1), supporting the hypothesis that increased DNM2 levels lead in part to the CNM phenotypes. No significant increase was observed in Dnm2 mRNA in $M t m 1^{-/ y}$ muscle, indicating that the increase in DNM2 protein expression is due to either increased stability of the protein or reduced degradation. While genetic deletion of a $D n m 2$ allele led to a $50 \%$ decrease at the protein level in $\mathrm{Dnm} 2^{+/-}$mice, we noted different DNM2 levels depending on the muscles investigated, following the same genetic deletion in the $M t m 1^{-/ y}$ mice. DNM2 protein levels were monitored at 50\% of WT levels in the diaphragm (Figure 2C) and $70 \%$ in TA muscles of $M t m 1^{-/ y} \mathrm{Dnm} 2^{+/-}$mice at 16 weeks (Figure 2E), and between $130 \%$ and $170 \%$ in $\mathrm{Mtm}^{-/ y} \mathrm{Dnm} 2^{\text {skm+/- }}$ and $M t m 1^{-/ y} \mathrm{Dnm} 2^{(i) s k m+/-}$ TA muscles (Figures 8 and 9). Importantly, the DNM2 levels correlated with the alteration of these muscles, as assessed by histology. As Mtm $1^{-/ y}$ mice died before this age, data for this genotype were not available, but we noted a consistent DNM2 increase in all muscles tested in 8-week-old $M t m 1^{-/ y}$ mice. Thus these data sustain the hypothesis that increased DNM2 levels led in part to the CNM phenotype and that decrease or normalization of DNM2 levels rescues the XLCNM phenotype. 
It was recently shown that increased flexibility of dynamin together with the tilting of the membrane-inserted pleckstrin homology $(\mathrm{PH})$ domain is a prerequisite for normal membrane fission (39). In ADCNM, DNM2 mutations are mostly clustered at the interface between the middle and $\mathrm{PH}$ domains $(24,40)$, suggesting they affect the stability of a closed conformation and lead to increased flexibility of this region, tilting of the $\mathrm{PH}$ domain, and membrane fission (24). Overactivation of dynamin-mediated membrane fission either by mutations or increased DNM2 levels may thus alter the proper balance for membrane remodeling. While T-tubule defects are a hallmark of CNM and thus regulation of membrane tubulation at this site a tempting hypothesis for explaining the MTM1/DNM2 pathway, dynamin was never observed on T-tubules in normal adult muscle $(28,29)$. At the physiological level, decreased DNM2 strongly reduced or delayed signs of CNM in $\mathrm{Mtm1}^{-1 / y}$ mice, including muscle mass, maximal force, and triad and nuclei positioning. This indicates a role for endogenous DNM2 in these different processes. Future studies will need to address the site of action of DNM2 in muscle.

$D N M 2$ reduction as a potential therapy for XLCNM. We identify here a cross-therapy strategy, whereby we downregulate a CNM gene (DNM2) to rescue the severe phenotype due to a downregulation of another CNM gene (MTM1). This technique differs from therapeutic strategies that aim to compensate for the lack of a protein by reexpression of the corresponding gene or overexpression of a homolog or by modification of the mutated gene to create a functional protein (such as exon skipping).

A main goal of this study was to validate a rescue approach for XLCNM. We first reduced DNM2 levels during embryogenesis in all tissues of the $\mathrm{Mtm}^{-/ y}$ mice; this experimental setup led to an extensive delay in the appearance of CNM phenotypes, a strong amelioration of motor behavior, and a normal life span, while $M t m 1^{-1 y}$ mice died mostly in the first 2 months from progressive muscle weakness.

Both total and muscle-specific Mtm1- $/ y$ induced CNM phenotypes in mice, indicating XLCNM arises from a primary defect in skeletal muscle (10). We obtained a phenotypic rescue of XLCNM in $M t m 1^{-1 y}$ mice by reducing DNM2 levels specifically in skeletal muscle, supporting the concept that XLCNM derives from a primary skeletal muscle defect and that specific treatment of muscle will be enough to ameliorate this disease in humans. In addition, our rescue data strongly support that rescuing myofiber intracellular organization, including myofibril organization, triad structure, and to some extent, nuclei positioning has a direct beneficial outcome on muscle function and life span and sustain the idea that these defects are primary causes of the XLCNM pathology.

However, muscle-restricted downregulation of DNM2 from embryonic stages or from 3-week-old mice rescued only $70 \%-75 \%$ of mice, compared with $100 \%$ rescue of the $\mathrm{Mtm}^{-1 / y} \mathrm{Dnm} 2^{+/-}$mice. Muscle-specific knockdown of DNM2 may not be sufficient to rescue all $M t m 1^{-1 y}$ mice if other organs are also affected. The HSA promoter is expressed differently in different fiber types, with highest expression in type IIB fibers (41), whereas a key feature of CNM is increased type 1 fibers, which may account for the reduced survival rate.

Unlike the $M t m 1^{-1 y}$ mice, in which motor behavior of neonates appears normal and deteriorates from 2 to 3 weeks of age, followed by a rapid progressive muscle weakness, patients are born with a severe hypotonia and muscle weakness that do not appear progressive. In addition, several reports indicate decreased fetal movements. Using time-inducible heterozygous deletion of DNM2, we found decreasing DNM2 expression after birth and after the appearance of some CNM symptoms was sufficient to improve life span and muscle weight in $\mathrm{Mtm}^{-/ y}$ mice. Thus, decreasing DNM2 levels after birth appears sufficient to revert the deterioration of muscle histology and progression of the disease. This is indeed an important advantage for potential application of similar strategies in patients. To translate this proof-of-concept toward clinical trials, targeted downregulation of DNM2 or drugs inhibiting DNM2 function will have to be validated. Compounds inhibiting the function of DNM2 may be valuable for this aim, assuming their delivery and biodisponibility in skeletal muscle is effective. In conclusion, we have identified DNM2 as a potential therapeutic target for XLCNM.

\section{Methods}

An expanded Methods section can be found in the Supplemental Methods.

\section{Generation of Dnm2 beterozygous mice}

The targeting vector was created with LoxP sites flanking exon 8 of Dnm2 (Supplemental Figure 1), then linearized, and electroporated into ES cells. Recombinant ES cells were injected into C57BL/ 6 blastocysts that were implanted in pseudopregnant females and germline transmission determined. Mice bred and analyzed were 129 pas strain (CMV promoter). HSA-Cre C57BL/ 6 and HSA Cre-ER ${ }^{\mathrm{T} 2}$ mice were a kind gift from Daniel Metzger $(\operatorname{IGBMC})(37,38)$ and were used to produce mice with musclespecific reduction of DNM2.

\section{Generation of Mtm1-/yDnm2 heterozygous mice}

Female $\mathrm{Mtm1}^{+/-}$mice (129pas strain) $(10,15)$ were bred with male Dnm2 heterozygous mice to produce 4 possible genotypes in male offspring: $M t m 1^{+/ y} \mathrm{Dnm}^{+/+}(\mathrm{WT}) ; \mathrm{Mtm}^{+/ y} \mathrm{Dnm} 2^{+/-}\left(\mathrm{Dnm}^{+/-}\right) ; \mathrm{Mtm1}^{-/ y} \mathrm{Dnm2} 2^{+/+}\left(\mathrm{Mtm1}^{-/ y}\right)$; and $M t m 1^{-/ y} \mathrm{Dmm}^{+/-}$. All mice analyzed in this study were male.

\section{Animal experiments}

Animals were housed in a temperature-controlled room $\left(19-22^{\circ} \mathrm{C}\right)$ with a 12-hour light/12-hour dark cycle. Mice were humanely sacrificed by $\mathrm{CO}_{2}$ inhalation followed by cervical dislocation, according to national and European legislation on animal experimentation. Muscles and other tissues were dissected and frozen in nitrogen-cooled isopentane and liquid nitrogen for histological and immunoblot assays, respectively.

\section{Phenotyping of Dnm2 ${ }^{+/-}$mice}

Dnm2 $2^{+/-}$male and female mice aged 10 to 15 weeks were phenotyped under the EUMODIC phenotyping program (see Supplemental Methods).

\section{String, grip, hanging, rotarod, and footprint tests}

String test. Mice were suspended on a wire by their forelimbs and allowed 20 seconds to climb, bringing their hind limbs onto the wire.

Grip test. Maximal strength measured from 2 or 4 paws using a dynamometer (Bioseb).

Hanging test. Mice were suspended from a cage lid for a maximum of 60 seconds, and the time the mouse fell off the cage was recorded.

Rotarod test. Coordination and whole-body muscle strength and fatigability were tested using an accelerated rotating rod test (Panlab).

Footprint test. The angle of the hind limbs was measured by analyzing the footprint pattern using the ImageJ analysis program ( $n=5-8$ mice per group).

\section{Plethysmograph measurements}

The spontaneous breathing pattern in nonrestrained unstimulated mice was measured using a whole-body barometric plethysmograph (EMKA Technologies) at the Institut Clinique de la Souris (ICS) (Illkirch, France) $(n=3-5$ mice per group). 


\section{TA and diaphragm muscle contractile properties}

TA muscle force measurements were evaluated by measuring in situ muscle isometric contraction in response to nerve and muscle stimulation ( $n=5-11$ mice per group). Isometric contraction was assessed on muscle strips from the ventral part of the costal diaphragm ( $n=3-5$ mice per group).

\section{Western blotting}

Western blotting was performed as described previously (28). Primary antibodies were as follows: home-made DNM2-R2680 (1:500), DNM2-R2865 (1:500), MTM1-R2827 (1:500), and GAPDH (1:10,000, MAB374; Chemicon); secondary antibodies were as follows: anti-rabbit HRP or anti-mouse HRP (1:10,000, Jackson ImmunoResearch Laboratories).

\section{qRT-PCR}

Total RNA was extracted from 8-week-old TA muscle lysates using Tri reagent (Molecular Research Center), reverse transcribed, and amplified using Oligo dT primers. Real-time qRT-PCR was performed using a Lightcycler 480 (Roche Diagnostics) with DNM2 primers (forward, CCAACAAAGGCATCTCCCCT; reverse, TGGTGAGTAGACCCGAAGGT) and GAPDH primers as a standard, with the SYBR Green 1 Master kit (Roche Diagnostics). Results were standardized to GAPDH $(n=2-3$ mice per group, performed in triplicate).

\section{Histology and immunofluorescence}

Longitudinal and transverse cryosections $(8 \mu \mathrm{m})$ of mouse skeletal muscles were prepared, fixed, and stained with the indicated antibodies and viewed using a laser-scanning confocal microscope (TCS SP5; Leica Microsystems). H\&E and SDH sections were imaged with a slide scanner NanoZoomer 2HT equipped with the fluorescence module L11600-21 (Hamamatsu Photonics). Cross-sectional area (CSA) and nuclei position were analyzed in $\mathrm{H} \& \mathrm{E}$ sections from TA mouse skeletal muscle, using FIJI image analysis software ( $n=4-7$ mice per group).

\section{TEM}

TEM was performed on TA muscle biopsies as described previously $(10,28)$. The ratio of triads to sarcomere was calculated as described previously (34).

\section{Microscopy and statistical analysis}

All microscopy was performed at the IGBMC Imaging Centre. All samples for microscopy were mounted in Fluorsave reagent (Merck). Light microscopy was performed using a fluorescence microscope (DM4000; Leica Microsystems) fitted with a color CCD camera (CoolSNAP cf color; Photometrics). Confocal microscopy was performed using a confocal laserscanning microscope (TCS SP2 or SP5; Leica Microsystems). ImageJ and FIJI analysis software were used for image analysis. Statistical analysis was performed using the unpaired Student's $t$ test (2-tailed) unless stated otherwise. $P<0.05$ was considered significant.

\section{Study approval}

Animal experimentation was approved by the institutional ethical committee Com'Eth IGBMC-ICS (2012-128). All human biopsies were taken after informed consent was obtained. The use of patient biopsies for this study was authorized by the ethical committee of Pitié-Salpêtrière Hospital (CCPPRB).

\section{Acknowledgments}

We thank Julien Becker, Jean-Luc Weickert, Olivia Wendling, Marc Koch,and Pascal Kessler for excellent technical assistance (IGBMC), and the animal house, histology platform, and imaging centre of the IGBMC and ICS for support. We thank Daniel Metzger (IGBMC) for helpful discussion, comments, and critical reading of the manuscript. This study was supported by INSERM, CNRS, University of Strasbourg (UdS), Collège de France, Fondation pour la Recherche Médicale (FRM; DEQ20071210538), and the E-rare program (no. 11-040). B.S. Cowling was supported by an FRM postdoctoral fellowship.

Received for publication May 24, 2013, and accepted in revised form November 21, 2013.

Address correspondence to: Jocelyn Laporte, IGBMC, 1 rue Laurent Fries, BP10142, 67404 Illkirch, France. Phone: 33388653412; Fax: 33388653201; E-mail: jocelyn@igbmc.fr.
1. Jungbluth H, Wallgren-Pettersson C, Laporte J. Centronuclear (myotubular) myopathy. Orphanet J Rare Dis. 2008;3:26.

2. Romero NB, Bitoun M. Centronuclear myopathies. Semin Pediatr Neurol. 2011;18(4):250-256.

3. Laporte J, et al. A gene mutated in X-linked myotubular myopathy defines a new putative tyrosine phosphatase family conserved in yeast. Nat Genet. 1996;13(2):175-182.

4. Nicot AS, et al. Mutations in amphiphysin 2 (BIN1) disrupt interaction with dynamin 2 and cause autosomal recessive centronuclear myopathy. Nat Genet. 2007;39(9):1134-1139.

5. Bitoun $\mathrm{M}$, et al. Mutations in dynamin 2 cause dominant centronuclear myopathy. Nat Genet. 2005;37(11):1207-1209.

6. Laporte J, Kress W, Mandel JL. Diagnosis of $\mathrm{X}$-linked myotubular myopathy by detection of myotubularin. Ann Neurol. 2001;50(1):42-46.

7. Laporte J, et al. MTM1 mutations in X-linked myotubular myopathy. Hum Mutat. 2000;15(5):393-409.

8. Tsai TC, et al. Characterization of MTM1 mutations in 31 Japanese families with myotubular myopathy, including a patient carrying $240 \mathrm{~kb}$ deletion in Xq28 without male hypogenitalism. Neuromuscul Disord. 2005;15(3):245-252.

9. Cowling BS, Toussaint A, Muller J, Laporte J. Defective membrane remodeling in neuromuscular diseases: insights from animal models. PLoS Genet. 2012;8(4):e1002595.
10. Buj-Bello A, et al. The lipid phosphatase myotubularin is essential for skeletal muscle maintenance but not for myogenesis in mice. Proc Natl Acad Sci U S A. 2002;99(23):15060-15065.

11. Pierson CR, et al. Modeling the human MTM1 p.R69C mutation in murine Mtm1 results in exon 4 skipping and a less severe myotubular myopathy phenotype. Hum Mol Genet. 2012;21(4):811-825.

12. Fetalvero KM, et al. Defective autophagy and mTORC1 signaling in myotubularin null mice. Mol Cell Biol. 2013;33(1):98-110.

13. Toussaint A, et al. Defects in amphiphysin 2 (BIN1) and triads in several forms of centronuclear myopathies. Acta Neuropathol. 2011;121(2):253-266.

14. Dowling JJ, et al. Loss of myotubularin function results in T-tubule disorganization in zebrafish and human myotubular myopathy. PLoS Genet. 2009;5(2):e1000372.

15. Al-Qusairi L, et al. T-tubule disorganization and defective excitation-contraction coupling in muscle fibers lacking myotubularin lipid phosphatase. Proc Natl Acad Sci U S A. 2009;106(44):18763-18768.

16. Amoasii L, et al. Myotubularin and PtdIns3P remodel the sarcoplasmic reticulum in muscle in vivo. J Cell Sci. 2013;126(pt 8):1806-1819.

17. Jones SM, Howell KE, Henley JR, Cao H, McNiven MA. Role of dynamin in the formation of transport vesicles from the trans-Golgi network. Science. 1998;279(5350):573-577

18. van der Bliek AM, Meyerowitz EM. Dynamin-like protein encoded by the Drosophila shibire gene associated with vesicular traffic. Nature. 1991; 351(6325):411-414.

19. Praefcke GJ, McMahon HT. The dynamin superfamily: universal membrane tubulation and fission molecules? Nat Rev Mol Cell Biol. 2004;5(2):133-147.

20. Ferguson SM, De Camilli P. Dynamin, a membrane-remodelling GTPase. Nat Rev Mol Cell Biol. 2012;13(2):75-88.

21. McNiven MA, Kim L, Krueger EW, Orth JD, Cao H, Wong TW. Regulated interactions between dyna$\mathrm{min}$ and the actin-binding protein cortactin modulate cell shape. J Cell Biol. 2000;151(1):187-198.

22. Takei K, McPherson PS, Schmid SL, De Camilli P. Tubular membrane invaginations coated by dynamin rings are induced by GTP-gamma $S$ in nerve terminals. Nature. 1995;374(6518):186-190.

23. Roux A, Uyhazi K, Frost A, De Camilli P. GTPdependent twisting of dynamin implicates constriction and tension in membrane fission. Nature. 2006;441(7092):528-531.

24. Faelber K, et al. Crystal structure of nucleotide-free dynamin. Nature. 2011;477(7366):556-560.

25. Zuchner S, et al. Mutations in the pleckstrin homology domain of dynamin 2 cause dominant intermediate Charcot-Marie-Tooth disease. Nat Genet. 2005;37(3):289-294.

26. Wang L, Barylko B, Byers C, Ross JA, Jameson DM, Albanesi JP. Dynamin 2 mutants linked to centronuclear myopathies form abnormally stable poly- 
mers. J Biol Chem. 2010;285(30):22753-22757.

27. Kenniston JA, Lemmon MA. Dynamin GTPase regulation is altered by $\mathrm{PH}$ domain mutations found in centronuclear myopathy patients. EMBO J. 2010;29(18):3054-3067.

28. Cowling BS, et al. Increased expression of wild-type or a centronuclear myopathy mutant of dynamin 2 in skeletal muscle of adult mice leads to structural defects and muscle weakness. Am J Pathol. 2011;178(5):2224-2235.

29. Durieux AC, et al. A centronuclear myopathydynamin 2 mutation impairs skeletal muscle structure and function in mice. Hum Mol Genet. 2010;19(24):4820-4836.

30. Liu N, et al. Mice lacking microRNA 133a develop dynamin 2-dependent centronuclear myopathy. J Clin Invest. 2011;121(8):3258-3268.

31. Ferguson SM, et al. Coordinated actions of actin and BAR proteins upstream of dynamin at endocytic clathrin-coated pits. Dev Cell. 2009;17(6):811-822.

32. Ayadi A, et al. Mouse large-scale phenotyping initiatives: overview of the European Mouse Disease Clinic (EUMODIC) and of the Wellcome Trust Sanger Institute Mouse Genetics Project. Mamm Genome. 2012;23(9-10):600-610.

33. Hnia K, et al. Myotubularin controls desmin intermediate filament architecture and mitochondrial dynamics in human and mouse skeletal muscle. J Clin Invest. 2011;121(1):70-85.

34. Amoasii L, et al. Phosphatase-dead myotubularin ameliorates $x$-linked centronuclear myopathy phenotypes in mice. PLoS Genet. 2012;8(10):e1002965.

35. Beggs AH, et al. MTM1 mutation associated with X-linked myotubular myopathy in Labrador retrievers. Proc Natl Acad Sci U S A. 2010; 107(33):14697-14702.

36. Al-Qusairi L, Laporte J. T-tubule biogenesis and triad formation in skeletal muscle and implication in human diseases. Skelet Muscle. 2011;1(1):26

37. Schuler M, Ali F, Metzger E, Chambon P, Metzger D. Temporally controlled targeted somatic mutagenesis in skeletal muscles of the mouse. Genesis. 2005;41(4):165-170.

38. Miniou P, Tiziano D, Frugier T, Roblot N, Le Meur $\mathrm{M}$, Melki J. Gene targeting restricted to mouse striated muscle lineage. Nucleic Acids Res. 1999;27(19):e27.

39. Shnyrova AV, et al. Geometric catalysis of membrane fission driven by flexible dynamin rings. Science. 2013;339(6126):1433-1436.

40. Bohm J, et al. Mutation spectrum in the large GTPase dynamin 2, and genotype-phenotype correlation in autosomal dominant centronuclear myopathy. Hum Mutat. 2012;33(6):949-959.

41. Tinsley JM, Potter AC, Phelps SR, Fisher R, Trickett JI, Davies KE. Amelioration of the dystrophic phenotype of mdx mice using a truncated utrophin transgene. Nature. 1996;384(6607):349-353. 